\title{
An efficient solver for space-time isogeometric Galerkin methods for parabolic problems
}

\author{
Gabriele Loli ${ }^{\mathrm{a}}$, Monica Montardini ${ }^{\mathrm{a}, *}$, Giancarlo Sangalli ${ }^{\mathrm{a}, \mathrm{b}}$, Mattia Tani $^{\mathrm{b}}$ \\ ${ }^{a}$ Dipartimento di Matematica "F. Casorati", Università di Pavia, Via A. Ferrata, 5, 27100 Pavia, Italy. \\ ${ }^{b}$ Istituto di Matematica Applicata e Tecnologie Informatiche, "E. Magenes" del CNR, Via A. Ferrata, 1, 27100 Pavia, Italy.
}

\begin{abstract}
In this work we focus on the preconditioning of a Galerkin space-time isogeometric discretization of the heat equation. Exploiting the tensor product structure of the basis functions in the parametric domain, we propose a preconditioner that is the sum of Kronecker products of matrices and that can be efficiently applied thanks to an extension of the classical Fast Diagonalization method. The preconditioner is robust w.r.t. the polynomial degree of the spline space and the time required for the application is almost proportional to the number of degrees-of-freedom, for a serial execution. By incorporating some information on the geometry parametrization and on the equation coefficients, we keep high efficiency with non-trivial domains and variable thermal conductivity and heat capacity coefficients.
\end{abstract}

Keywords: Isogeometric Analysis, splines, heat equation, space-time Galerkin formulation, Fast Diagonalization.

\section{Introduction}

Isogeometric Analysis (IgA), introduced in the seminal paper [1] (see also the book 2]), is an evolution of the classical finite element methods. IgA uses spline functions, or their generalizations, both to represent the computational domain and to approximate the solution of the partial differential equation that models the problem of interest. This is meant to simplify the interoperability between computer aided design and numerical simulations. IgA also benefits from the approximation properties of splines, whose high-continuity yields higher accuracy when compared to $C^{0}$ piecewise polynomials, see e.g., [3, 4, 5].

In this paper we focus on the heat equation and on its space-time Galerkin isogeometric discretization. Space-time finite element methods originated in the papers [6, 7, 8, where standard finite elements are ascribed an extra dimension for the time and, typically, adopt a discontinuous approximation in time, since this produces a time marching algorithm with a traditional step-by-step format (see e.g. 9]).

One of the first work concerning space-time isogeometric discretization is [10, in which a stabilized variational formulation produces a discrete bilinear form that is elliptic with respect to a discrete energy norm. The resulting linear system is then solved through a standard parallel AMG preconditioned GMRES solver. Other papers in literature propose isogeometric space-time Galerkin methods, favoring a step-bystep structure in time. In 11, the same variational formulation of 10 is used in combination with a space-time domain decomposition into space-time slabs that are sequentially coupled in time by a stabilized discontinuous Galerkin method. In [12, two different methods, called ST-C-SPT and ST-C-DCT, are outlined. The first one, analysed in [13, is a way to project a previously computed solution, possibly discontinuous, into isogeometric spaces in order to get a more regular solution and to save memory for

\footnotetext{
* Corresponding author

Email addresses: gabriele.loli01@universitadipavia.it (Gabriele Loli), monica.montardini01@universitadipavia.it (Monica Montardini), giancarlo.sangalli@unipv.it (Giancarlo Sangalli), mattia.tani@imati.cnr.it (Mattia Tani)
} 
its storage. In the ST-C-DCT method, the solution with continuous temporal representation is computed sequentially from the space-time variational formulation associated with each slab.

Related multigrid solvers have been proposed in [14, 15] and low-rank approximations in [16. In [17] the authors consider $C^{0}$ coupling between the space-time slabs with a suitable stabilized formulation that also yields a sequential scheme. Finally, the interest in space-time isogeometric analysis for complex realworld simulations is attested by the recent papers [18, 19, 20], where, again, a sequential (discontinuous) approximation in time is adopted.

The novelty of our work is that we deal with smooth approximation in both space and time. This started in a previous work, 21, based on a $L^{2}$ least-squares formulation. The reason of this choice is that the problem becomes elliptic and a preconditioner for the linear system can be easily designed as in 22 . Indeed, when adopting smooth approximation in space and in time, the major issue is its computational cost and the key ingredient is an efficient solver for the linear system, which is global in time. In the present work, instead, we focus on the plain Galerkin space-time formulation, whose well-posedness has been studied, for finite element discretizations and for the heat equation, in the recent papers [23] and [24]. For a Galerkin formulation, and assuming that the spatial domain does not change with time, the linear system has the structure

$$
\gamma \mathbf{W}_{t} \otimes \mathbf{M}_{s}+\nu \mathbf{M}_{t} \otimes \mathbf{K}_{s}
$$

where $\mathbf{W}_{t}$ is given by the discretization of the time derivative, $\mathbf{K}_{s}$ is given by the discretization of the Laplacian in the spatial variables, $\mathbf{M}_{t}$ and $\mathbf{M}_{s}$ are "mass matrices" in time and space, respectively, and $\gamma, \nu>0$ are constants of the problem. Adopting an iterative solver, we do not need to form the matrix (1.1) (observe that the cost of formation of the matrices in 1.1 is comparable to the cost of forming a steady-state diffusion matrix) but there is the need of an efficient preconditioning strategy. The main contribution of this paper is the construction of a preconditioner for (1.1) generalizing the classical Fast Diagonalization (FD) method [25. Indeed, the FD method cannot be directly applied to (1.1), as this would require to compute the eigendecomposition of the pencil $\left(\mathbf{W}_{t}, \mathbf{M}_{t}\right)$ which is numerically unstable. We circumvent this difficulty by introducing an ad-hoc factorization of the time matrices which allows to design a solver conceptually similar to the FD method. The computational cost of the setup of the resulting preconditioner is $O\left(N_{d o f}\right)$ FLOating-Point operations (FLOPs) while its application is $O\left(N_{d o f}^{1+1 / d}\right)$ FLOPs, where $d$ is the number of spatial dimensions and $N_{d o f}$ denotes the total number of degrees-of-freedom (assuming, for simplicity, to have the same number of degrees-of-freedom in time and in each spatial direction). Our numerical benchmarks show that the computing time (serial and single-core execution) is close to optimality, that is, proportional to $N_{d o f}$. The preconditioner is also robust with respect to the polynomial degree. Furthermore, our approach is optimal in terms of memory requirement: denoting by $N_{s}$ the total number of degrees-of-freedom in space, the storage cost is $O\left(p^{d} N_{s}+N_{d o f}\right)$. We also remark that global space-time methods in principle facilitate the full parallelization of the solver, see [26, 27, 28.

The outline of the paper is as follows. In Section 2 we present the basics of B-splines based IgA and the main properties of the Kronecker product operation. The model problem and its isogeometric discretization are introduced in Section 3, while in Section 4 we define the preconditioner and we discuss its application. We present the numerical results assessing the performance of the proposed preconditioner in Section 5 . Finally, in the last section we draw some conclusions and we highlight some future research directions.

\section{Preliminaries}

\subsection{B-Splines}

Given $m$ and $p$ two positive integers, a knot vector in $[0,1]$ is a sequence of non-decreasing points $\Xi:=\left\{0=\xi_{1} \leq \cdots \leq \xi_{m+p+1}=1\right\}$. We consider open knot vectors, i.e. we set $\xi_{1}=\cdots=\xi_{p+1}=0$ and $\xi_{m}=\cdots=\xi_{m+p+1}=1$. Then, according to Cox-De Boor recursion formulas (see [29]), univariate B-splines $\widehat{b}_{i, p}:(0,1) \rightarrow \mathbb{R}$ are piecewise polynomials defined for $i=1, \ldots, m$ as 
for $p=0$ :

$$
\widehat{b}_{i, 0}(\eta)= \begin{cases}1 & \text { if } \xi_{i} \leq \eta<\xi_{i+1} \\ 0 & \text { otherwise }\end{cases}
$$

for $p \geq 1$ :

$$
\widehat{b}_{i, p}(\eta)= \begin{cases}\frac{\eta-\xi_{i}}{\xi_{i+p}-\xi_{i}} \widehat{b}_{i, p-1}(\eta)+\frac{\xi_{i+p+1}-\eta}{\xi_{i+p+1}-\xi_{i+1}} \widehat{b}_{i+1, p-1}(\eta) & \text { if } \xi_{i} \leq \eta<\xi_{i+p+1}, \\ 0 & \text { otherwise }\end{cases}
$$

where we adopt the convention $0 / 0=0$. The univariate spline space is defined as

$$
\widehat{\mathcal{S}}_{h}^{p}:=\operatorname{span}\left\{\widehat{b}_{i, p}\right\}_{i=1}^{m},
$$

where $h$ denotes the mesh-size, i.e. $h:=\max \left\{\left|\xi_{i+1}-\xi_{i}\right| \mid i=1, \ldots, m+p\right\}$. The interior knot multiplicity influences the smoothness of the B-splines at the knots (see 29]). For more details on B-splines properties and their use in IgA we refer to [2].

Multivariate B-splines are defined as tensor product of univariate B-splines. We consider functions that depend on $d$ spatial variables and the time variable. Therefore, given positive integers $m_{l}, p_{l}$ for $l=1, \ldots, d$ and $m_{t}, p_{t}$, we introduce $d+1$ univariate knot vectors $\Xi_{l}:=\left\{\xi_{l, 1} \leq \cdots \leq \xi_{l, m_{l}+p_{l}+1}\right\}$ for $l=1, \ldots, d$ and $\Xi_{t}:=\left\{\xi_{t, 1} \leq \cdots \leq \xi_{t, m_{t}+p_{t}+1}\right\}$. Let $h_{l}$ be the mesh-size associated to the knot vector $\Xi_{l}$ for $l=1, \ldots, d$, let $h_{s}:=\max \left\{h_{l} \mid l=1, \ldots, d\right\}$ be the maximal mesh-size in all spatial knot vectors and let $h_{t}$ be the mesh-size of the time knot vector. Let also $\boldsymbol{p}$ be the vector that contains the degree indexes, i.e. $\boldsymbol{p}:=\left(\boldsymbol{p}_{s}, p_{t}\right)$, where $\boldsymbol{p}_{s}:=\left(p_{1}, \ldots, p_{d}\right)$. For simplicity, we assume to have the same polynomial degree in all spatial directions, i.e., with abuse of notations, we set $p_{1}=\cdots=p_{d}=: p_{s}$, but the general case is similar.

We assume that the following quasi-uniformity of the knot vectors holds.

Assumption 1. There exists $0<\alpha \leq 1$, independent of $h_{s}$ and $h_{t}$, such that each non-empty knot span $\left(\xi_{l, i}, \xi_{l, i+1}\right)$ of $\Xi_{l}$ fulfils $\alpha h_{s} \leq \xi_{l, i+1}-\xi_{l, i} \leq h_{s}$ for $l=1, \ldots, d$ and each non-empty knot-span $\left(\xi_{t, i}, \xi_{t, i+1}\right)$ of $\Xi_{t}$ fulfils $\alpha h_{t} \leq \xi_{t, i+1}-\xi_{t, i} \leq h_{t}$.

The multivariate B-splines are defined as

$$
\widehat{B}_{i, \boldsymbol{p}}(\boldsymbol{\eta}, \tau):=\widehat{B}_{\boldsymbol{i}_{\boldsymbol{s}}, \boldsymbol{p}_{s}}(\boldsymbol{\eta}) \widehat{b}_{i_{t}, p_{t}}(\tau),
$$

where

$$
\widehat{B}_{\boldsymbol{i}_{s}, \boldsymbol{p}_{s}}(\boldsymbol{\eta}):=\widehat{b}_{i_{1}, p_{s}}\left(\eta_{1}\right) \ldots \widehat{b}_{i_{d}, p_{s}}\left(\eta_{d}\right),
$$

$\boldsymbol{i}_{\boldsymbol{s}}:=\left(i_{1}, \ldots, i_{d}\right), \boldsymbol{i}:=\left(\boldsymbol{i}_{\boldsymbol{s}}, i_{t}\right)$ and $\boldsymbol{\eta}=\left(\eta_{1}, \ldots, \eta_{d}\right)$. The corresponding spline space is defined as

$$
\widehat{\mathcal{S}}_{h}^{p}:=\operatorname{span}\left\{\widehat{B}_{\boldsymbol{i}, \boldsymbol{p}} \mid i_{l}=1, \ldots, m_{l} \text { for } l=1, \ldots, d ; i_{t}=1, \ldots, m_{t}\right\},
$$

where $h:=\max \left\{h_{s}, h_{t}\right\}$. We have that $\widehat{\mathcal{S}}_{h}^{p}=\widehat{\mathcal{S}}_{h_{s}}^{p_{s}} \otimes \widehat{\mathcal{S}}_{h_{t}}^{p_{t}}$, where

$$
\widehat{\mathcal{S}}_{h_{s}}^{\boldsymbol{p}_{s}}:=\operatorname{span}\left\{\widehat{B}_{\boldsymbol{i}_{s}, \boldsymbol{p}_{s}} \mid i_{l}=1, \ldots, m_{l} ; l=1, \ldots, d\right\}
$$

is the space of tensor-product splines on $\widehat{\Omega}:=(0,1)^{d}$.

Assumption 2. We assume that $p_{t}, p_{s} \geq 1$ and that $\widehat{\mathcal{S}}_{h_{s}}^{p_{s}} \subset C^{0}(\widehat{\Omega})$ and $\widehat{\mathcal{S}}_{h_{t}}^{p_{t}} \subset C^{0}((0,1))$. 


\subsection{Isogeometric spaces}

The space-time computational domain that we consider is $\Omega \times(0, T)$, where $\Omega \subset \mathbb{R}^{d}$ and $T>0$ is the final time. We make the following assumption.

Assumption 3. We assume that $\Omega$ is parametrized by $\boldsymbol{F}: \widehat{\Omega} \rightarrow \Omega$, with $\boldsymbol{F} \in\left[\widehat{\mathcal{S}}_{h_{s}}^{\boldsymbol{p}_{s}}\right]^{d}$. Moreover, we assume that $\boldsymbol{F}^{-1}$ has piecewise bounded derivatives of any order.

We define $\boldsymbol{x}=\left(x_{1}, \ldots, x_{d}\right):=\boldsymbol{F}(\boldsymbol{\eta})$ and $t:=T \tau$. Then the space-time domain is given by the parametrization $\boldsymbol{G}: \widehat{\Omega} \times(0,1) \rightarrow \Omega \times(0, T)$, such that $\boldsymbol{G}(\boldsymbol{\eta}, \tau):=(\boldsymbol{F}(\boldsymbol{\eta}), T \tau)=(\boldsymbol{x}, t)$.

We introduce the spline space with initial and boundary conditions, in parametric coordinates, as

$$
\widehat{\mathcal{X}}_{h}:=\left\{\widehat{v}_{h} \in \widehat{\mathcal{S}}_{h}^{p} \mid \widehat{v}_{h}=0 \text { on } \partial \widehat{\Omega} \times(0,1) \text { and } \widehat{v}_{h}=0 \text { on } \widehat{\Omega} \times\{0\}\right\} .
$$

We also have that $\widehat{\mathcal{X}}_{h}=\widehat{\mathcal{X}}_{s, h_{s}} \otimes \widehat{\mathcal{X}}_{t, h_{t}}$, where

$$
\begin{aligned}
\widehat{\mathcal{X}}_{s, h_{s}} & :=\left\{\widehat{w}_{h} \in \widehat{\mathcal{S}}_{h_{s}}^{p_{s}} \mid \widehat{w}_{h}=0 \text { on } \partial \widehat{\Omega}\right\} \\
& =\operatorname{span}\left\{\widehat{b}_{i_{1}, p_{s}} \ldots \widehat{b}_{i_{d}, p_{s}} \mid i_{l}=2, \ldots, m_{l}-1 ; l=1, \ldots, d\right\}, \\
\widehat{\mathcal{X}}_{t, h_{t}} & :=\left\{\widehat{w}_{h} \in \widehat{\mathcal{S}}_{h_{t}}^{p_{t}} \mid \widehat{w}_{h}(0)=0\right\}=\operatorname{span}\left\{\widehat{b}_{i_{t}, p_{t}} \mid i_{t}=2, \ldots, m_{t}\right\} .
\end{aligned}
$$

By introducing a colexicographical reordering of the basis functions, we can write

$$
\begin{aligned}
\widehat{\mathcal{X}}_{s, h_{s}} & =\operatorname{span}\left\{\widehat{b}_{i_{1}, p_{s}} \ldots \widehat{b}_{i_{d}, p_{s}} \mid i_{l}=1, \ldots, n_{s, l} ; l=1, \ldots, d\right\} \\
& =\operatorname{span}\left\{\widehat{B}_{i, p_{s}} \mid i=1, \ldots, N_{s}\right\}, \\
\widehat{\mathcal{X}}_{t, h_{t}} & =\operatorname{span}\left\{\widehat{b}_{i, p_{t}} \mid i=1, \ldots, n_{t}\right\},
\end{aligned}
$$

and then

$$
\widehat{\mathcal{X}}_{h}=\operatorname{span}\left\{\widehat{B}_{i, \boldsymbol{p}} \mid i=1, \ldots, N_{\text {dof }}\right\},
$$

where we defined $n_{s, l}:=m_{l}-2$ for $l=1, \ldots, d, N_{s}:=\prod_{l=1}^{d} n_{s, l}, n_{t}:=m_{t}-1$ and $N_{d o f}:=N_{s} n_{t}$.

Finally, the isogeometric space we consider is the isoparametric push-forward of $(2.3)$ through the geometric map $\boldsymbol{G}$, i.e.

$$
\mathcal{X}_{h}:=\operatorname{span}\left\{B_{i, \boldsymbol{p}}:=\widehat{B}_{i, \boldsymbol{p}} \circ \boldsymbol{G}^{-1} \mid i=1, \ldots, N_{d o f}\right\} .
$$

We also have that $\mathcal{X}_{h}=\mathcal{X}_{s, h_{s}} \otimes \mathcal{X}_{t, h_{t}}$, where

$$
\mathcal{X}_{s, h_{s}}:=\operatorname{span}\left\{B_{i, \boldsymbol{p}_{s}}:=\widehat{B}_{i, \boldsymbol{p}_{s}} \circ \boldsymbol{F}^{-1} \mid i=1, \ldots, N_{s}\right\}
$$

and

$$
\mathcal{X}_{t, h_{t}}:=\operatorname{span}\left\{b_{i, p_{t}}:=\widehat{b}_{i, p_{t}}(\cdot / T) \mid i=1, \ldots, n_{t}\right\} .
$$

\subsection{Kronecker product}

The Kronecker product of two matrices $\mathbf{C} \in \mathbb{C}^{n_{1} \times n_{2}}$ and $\mathbf{D} \in \mathbb{C}^{n_{3} \times n_{4}}$ is defined as

$$
\mathbf{C} \otimes \mathbf{D}:=\left[\begin{array}{ccc}
{[\mathbf{C}]_{1,1} \mathbf{D}} & \ldots & {[\mathbf{C}]_{1, n_{2}} \mathbf{D}} \\
\vdots & \ddots & \vdots \\
{[\mathbf{C}]_{n_{1}, 1} \mathbf{D}} & \ldots & {[\mathbf{C}]_{n_{1}, n_{2}} \mathbf{D}}
\end{array}\right] \in \mathbb{C}^{n_{1} n_{3} \times n_{2} n_{4}},
$$


where $[\mathbf{C}]_{i, j}$ denotes the $i j$-th entry of the matrix $\mathbf{C}$. For extensions and properties of the Kronecker product we refer to [30. In particular, when a matrix has a Kronecker product structure, the matrix-vector product can be efficiently computed. For this purpose, for $m=1, \ldots, d+1$ we introduce the $m$-mode product of a tensor $\mathfrak{X} \in \mathbb{C}^{n_{1} \times \cdots \times n_{d+1}}$ with a matrix $\mathbf{J} \in \mathbb{C}^{\ell \times n_{m}}$, that we denote by $\mathfrak{X} \times{ }_{m} \mathbf{J}$. This is a tensor of size $n_{1} \times \cdots \times n_{m-1} \times \ell \times n_{m+1} \times \ldots n_{d+1}$, whose elements are defined as

$$
\left[\mathfrak{X} \times_{m} \mathbf{J}\right]_{i_{1}, \ldots, i_{d+1}}:=\sum_{j=1}^{n_{m}}[\mathfrak{X}]_{i_{1}, \ldots, i_{m-1}, j, i_{m+1}, \ldots, i_{d+1}}[\mathbf{J}]_{i_{m}, j} .
$$

Then, given $\mathbf{J}_{i} \in \mathbb{C}^{\ell_{i} \times n_{i}}$ for $i=1, \ldots, d+1$, it holds

$$
\left(\mathbf{J}_{d+1} \otimes \cdots \otimes \mathbf{J}_{1}\right) \operatorname{vec}(\mathfrak{X})=\operatorname{vec}\left(\mathfrak{X} \times{ }_{1} \mathbf{J}_{1} \times_{2} \cdots \times_{d+1} \mathbf{J}_{d+1}\right),
$$

where the vectorization operator "vec" applied to a tensor stacks its entries into a column vector as

$$
[\operatorname{vec}(\mathfrak{X})]_{j}=[\mathfrak{X}]_{i_{1}, \ldots, i_{d+1}} \text { for } i_{l}=1, \ldots, n_{l} \text { and for } l=1, \ldots, d+1,
$$

where $j:=i_{1}+\sum_{k=2}^{d+1}\left[\left(i_{k}-1\right) \Pi_{l=1}^{k-1} n_{l}\right]$.

\section{The model problem}

\subsection{Space-time variational formulation}

Our model problem is the heat equation with homogeneous boundary and initial conditions: we look for a solution $u$ such that

$$
\left\{\begin{array}{rrrrr}
\gamma \partial_{t} u-\nabla \cdot(\nu \nabla u) & = & f & \text { in } & \Omega \times(0, T), \\
u & = & 0 & \text { on } & \partial \Omega \times[0, T] \\
u & = & 0 & \text { in } & \Omega \times\{0\}
\end{array}\right.
$$

where $\Omega \subset \mathbb{R}^{d}, T$ is the final time, $\gamma>0$ is the heat capacity constant and $\nu>0$ is the thermal conductivity constant. We assume that $f \in L^{2}\left(0, T ; H^{-1}(\Omega)\right)$ and we introduce the Hilbert spaces

$$
\begin{gathered}
\mathcal{X}:=\left\{v \in L^{2}\left(0, T ; H_{0}^{1}(\Omega)\right) \cap H^{1}\left(0, T ; H^{-1}(\Omega)\right) \mid v(\boldsymbol{x}, 0)=0\right\}, \\
\mathcal{Y}:=L^{2}\left(0, T ; H_{0}^{1}(\Omega)\right),
\end{gathered}
$$

endowed with the following norms

$$
\|v\|_{\mathcal{X}}^{2}:=\frac{\gamma^{2}}{\nu}\left\|\partial_{t} v\right\|_{L^{2}\left(0, T ; H^{-1}(\Omega)\right)}^{2}+\nu\|v\|_{L^{2}\left(0, T ; H_{0}^{1}(\Omega)\right)}^{2} \quad \text { and }\|v\|_{\mathcal{Y}}^{2}:=\nu\|v\|_{L^{2}\left(0, T ; H_{0}^{1}(\Omega)\right)}^{2}
$$

respectively. Then, the variational formulation of 3.1 reads:

$$
\text { Find } u \in \mathcal{X} \text { such that } \mathcal{A}(u, v)=\mathcal{F}(v) \quad \forall v \in \mathcal{Y},
$$

where the bilinear form $\mathcal{A}(\cdot, \cdot)$ and the linear form $\mathcal{F}(\cdot)$ are defined $\forall w \in \mathcal{X}$ and $\forall v \in \mathcal{Y}$ as

$$
\mathcal{A}(w, v):=\int_{0}^{T} \int_{\Omega}\left(\gamma \partial_{t} w v+\nu \nabla w \cdot \nabla v\right) \mathrm{d} \Omega \mathrm{dt} \quad \text { and } \quad \mathcal{F}(v):=\int_{0}^{T} \int_{\Omega} f v \mathrm{~d} \Omega \mathrm{dt} .
$$

The well-posedness of the variational formulation above is a classical result, see for example [23].

The previous setting can be generalized to non-homogeneous initial and boundary conditions. For example, suppose that in (3.1) we have the initial condition $u=u_{0}$ in $\Omega \times\{0\}$ with $u_{0} \in L^{2}(\Omega)$. Then, we consider a lifting $\underline{u}_{0}$ of $u_{0}$ such that $\underline{u}_{0} \in L^{2}\left(0, T ; H_{0}^{1}(\Omega)\right) \cap H^{1}\left(0, T ; H^{-1}(\Omega)\right)$, see e.g. 31. Finally, we split the solution $u$ as $u=\underline{u}+\underline{u}_{0}$, where $\underline{u} \in \mathcal{X}$ is the solution of the following heat equation with homogeneous initial and boundary conditions:

$$
\left\{\begin{array}{rrrrr}
\gamma \partial_{t} \underline{u}-\nabla \cdot(\nu \nabla \underline{u}) & = & & \text { in } & \Omega \times(0, T), \\
\underline{u} & = & 0 & \text { on } & \partial \Omega \times[0, T] \\
\underline{u} & = & 0 & \text { in } & \Omega \times\{0\}
\end{array}\right.
$$

where $\underline{f}:=f-\gamma \partial_{t} \underline{u}_{0}+\nabla \cdot\left(\nu \nabla \underline{u}_{0}\right)$. 


\subsection{Space-time Galerkin method} 3.2.:

Let $\mathcal{X}_{h} \subset \mathcal{X}$ be the isogeometric space defined in (2.4). We consider the following Galerkin method for

$$
\text { Find } u_{h} \in \mathcal{X}_{h} \text { such that } \mathcal{A}\left(u_{h}, v_{h}\right)=\mathcal{F}\left(v_{h}\right) \quad \forall v_{h} \in \mathcal{X}_{h} .
$$

Following [23, let $N_{h}: L^{2}\left(0, T ; H^{-1}(\Omega)\right) \rightarrow \mathcal{X}_{h}$ be the discrete Newton potential operator, defined as follows: given $\phi \in L^{2}\left(0, T ; H^{-1}(\Omega)\right)$ then $N_{h} \phi \in \mathcal{X}_{h}$ fulfills

$$
\int_{0}^{T} \int_{\Omega} \nu \nabla\left(N_{h} \phi\right) \cdot \nabla v_{h} \mathrm{~d} \Omega \mathrm{dt}=\gamma \int_{0}^{T} \int_{\Omega} \phi v_{h} \mathrm{~d} \Omega \mathrm{dt} \quad \forall v_{h} \in \mathcal{X}_{h}
$$

Thus, we define the norm in $\mathcal{X}_{h}$ as

$$
\|w\|_{\mathcal{X}_{h}}^{2}:=\nu\left\|N_{h}\left(\partial_{t} w\right)\right\|_{L^{2}\left(0, T ; H_{0}^{1}(\Omega)\right)}^{2}+\nu\|w\|_{L^{2}\left(0, T ; H_{0}^{1}(\Omega)\right)}^{2} .
$$

The stability and the well-posedness of formulation (3.3) are guaranteed by [23, Equation (2.7)] and by a straightforward extension to IgA of [23, Theorem 3.1] and [23, Theorem 3.2]. We summarize these results in the following Proposition 1 and Theorem 1.

Proposition 1. It holds

$$
\mathcal{A}(w, v) \leq \sqrt{2}\|w\|_{\mathcal{X}}\|v\|_{\mathcal{Y}} \quad \forall w \in \mathcal{X} \text { and } \forall v \in \mathcal{Y}
$$

and

$$
\left\|w_{h}\right\|_{\mathcal{X}_{h}} \leq 2 \sqrt{2} \sup _{v_{h} \in \mathcal{X}_{h}} \frac{\mathcal{A}\left(w_{h}, v_{h}\right)}{\left\|v_{h}\right\|_{\mathcal{Y}}} \quad \forall w_{h} \in \mathcal{X}_{h}
$$

Theorem 1. There exists a unique solution $u_{h} \in \mathcal{X}_{h}$ to the discrete problem (3.3). Moreover, it holds

$$
\left\|u-u_{h}\right\|_{\mathcal{X}_{h}} \leq 5 \inf _{w_{h} \in \mathcal{X}_{h}}\left\|u-w_{h}\right\|_{\mathcal{X}}
$$

where $u \in \mathcal{X}$ is the solution of 3.2 .

We have then the following a-priori estimate for $h$-refinement.

Theorem 2. Let $q$ be an integer such that $1<q \leq \min \left\{p_{s}, p_{t}\right\}+1$. If $u \in \mathcal{X} \cap H^{q}(\Omega \times(0, T))$ is the solution of 3.2 and $u_{h} \in \mathcal{X}_{h}$ is the solution of 3.3 , then it holds

$$
\left\|u-u_{h}\right\|_{\mathcal{X}_{h}} \leq C \sqrt{\frac{\gamma^{2}}{\nu}+\nu}\left(h_{t}^{q-1}+h_{s}^{q-1}\right)\|u\|_{H^{q}(\Omega \times(0, T))}
$$

where $C$ is independent of $h_{s}, h_{t}, \gamma, \nu$ and $u$.

Proof. We use the approximation estimates of the isogeometric spaces from [32]. We report here only the main steps, since the proof is similar to the one of [21, Proposition 4].

Let $\Pi_{h}: L^{2}(\Omega \times(0, T)) \rightarrow \mathcal{X}_{h}$ be a suitable projection, based on a tensor-product construction as in [32], and $L^{2}(\Omega \times(0, T)) \equiv L^{2}\left(0, T ; L^{2}(\Omega)\right) \equiv L^{2}(\Omega) \otimes L^{2}(0, T)$. Then $\Pi_{h}=\Pi_{s, h_{s}} \otimes \Pi_{t, h_{t}}$, where $\Pi_{s, h_{s}}$ : $L^{2}(\Omega) \rightarrow \mathcal{X}_{s, h_{s}}$ and $\Pi_{t, h_{t}}: L^{2}(0, T) \rightarrow \mathcal{X}_{t, h_{t}}$ are projections on the space and time components, respectively, of the isogeometric space $\mathcal{X}_{h}$. The following bounds are straightforward generalizations of [32, Proposition 4.1, Theorem 5.1]

$$
\begin{aligned}
\left\|\partial_{t}\left(u-\Pi_{h} u\right)\right\|_{L^{2}(\Omega) \otimes L^{2}(0, T)} & =\left|\left(u-\Pi_{h} u\right)\right|_{L^{2}(\Omega) \otimes H^{1}(0, T)} \\
& \leq C_{1}\left(h_{t}^{q-1}\|u\|_{L^{2}(\Omega) \otimes H^{q}(0, T)}+h_{s}^{q-1}\|u\|_{H^{q-1}(\Omega) \otimes H^{1}(0, T)}\right) \\
& \leq C_{1}\left(h_{t}^{q-1}+h_{s}^{q-1}\right)\|u\|_{H^{q}(\Omega \times(0, T))}
\end{aligned}
$$


and

$$
\begin{aligned}
\left\|u-\Pi_{h} u\right\|_{L^{2}\left(0, T ; H_{0}^{1}(\Omega)\right)} & =\left|u-\Pi_{h} u\right|_{H^{1}(\Omega) \otimes L^{2}(0, T)} \\
& \leq C_{2}\left(h_{t}^{q-1}\|u\|_{H^{1}(\Omega) \otimes H^{q-1}(0, T)}+h_{s}^{q-1}\|u\|_{H^{q}(\Omega) \otimes L^{2}(0, T)}\right) \\
& \leq C_{2}\left(h_{t}^{q-1}+h_{s}^{q-1}\right)\|u\|_{H^{q}(\Omega \times(0, T))}
\end{aligned}
$$

Therefore, using 3.5 with the obvious bound $\left\|\partial_{t}\left(u-\Pi_{h} u\right)\right\|_{L^{2}\left(0, T ; H^{-1}(\Omega)\right)} \leq\left\|\partial_{t}\left(u-\Pi_{h} u\right)\right\|_{L^{2}(\Omega) \otimes L^{2}(0, T)}$, and (3.6), we get

$$
\left\|u-\Pi_{h} u\right\|_{\mathcal{X}}^{2} \leq C_{3}\left(\frac{\gamma^{2}}{\nu}+\nu\right)\left(h_{t}^{q-1}+h_{s}^{q-1}\right)^{2}\|u\|_{H^{q}(\Omega \times(0, T))}^{2}
$$

and then (3.4), thanks to Theorem 11. The constants $C_{1}, C_{2}, C_{3}$ above are independent of $h_{s}, h_{t}, \gamma, \nu$ and $u$.

Remark 1. In Theorem 1, the degrees $p_{t}, p_{s}$ and the mesh-sizes $h_{t}, h_{s}$ play a similar role. This motivates our choice $p_{t}=p_{s}=: p$ and $h_{t}=h_{s}=: h$ for the numerical tests in Section 5. In this case, and if the solution $u$ is smooth, (3.4) yields h-convergence of order $p$. A sharper error analysis is possible taking into account a different regularity of the solution $u$ in space and time, in the line of the anisotropic estimates of [32].

\subsection{Discrete system}

The linear system associated to 3.3 is

$$
\mathbf{A u}=\mathbf{f},
$$

where $[\mathbf{A}]_{i, j}=\mathcal{A}\left(B_{j, \boldsymbol{p}}, B_{i, \boldsymbol{p}}\right)$ and $[\mathbf{f}]_{i}=\mathcal{F}\left(B_{i, \boldsymbol{p}}\right)$. The tensor-product structure of the isogeometric space (2.4) allows to write the system matrix $\mathbf{A}$ as sum of Kronecker products of matrices as

$$
\mathbf{A}=\gamma \mathbf{W}_{t} \otimes \mathbf{M}_{s}+\nu \mathbf{M}_{t} \otimes \mathbf{K}_{s}
$$

where for $i, j=1, \ldots, n_{t}$

$$
\left[\mathbf{W}_{t}\right]_{i, j}=\int_{0}^{T} b_{j, p_{t}}^{\prime}(t) b_{i, p_{t}}(t) \mathrm{dt} \quad \text { and } \quad\left[\mathbf{M}_{t}\right]_{i, j}=\int_{0}^{T} b_{i, p_{t}}(t) b_{j, p_{t}}(t) \mathrm{dt},
$$

while for $i, j=1, \ldots, N_{s}$

$$
\left[\mathbf{K}_{s}\right]_{i, j}=\int_{\Omega} \nabla B_{i, p_{s}}(\boldsymbol{x}) \cdot \nabla B_{j, p_{s}}(\boldsymbol{x}) \mathrm{d} \Omega \quad \text { and } \quad\left[\mathbf{M}_{s}\right]_{i, j}=\int_{\Omega} B_{i, p_{s}}(\boldsymbol{x}) B_{j, p_{s}}(\boldsymbol{x}) \mathrm{d} \Omega .
$$

\section{Preconditioner definition and application}

We introduce, for the system (3.8), the preconditioner

$$
[\widehat{\mathbf{A}}]_{i, j}:=\widehat{\mathcal{A}}\left(\widehat{B}_{j, \boldsymbol{p}}, \widehat{B}_{i, \boldsymbol{p}}\right),
$$

where

We have

$$
\widehat{\mathcal{A}}(\widehat{v}, \widehat{w}):=\int_{0}^{1} \int_{\widehat{\Omega}}\left(\gamma \partial_{t} \widehat{v} \widehat{w}+T \nu \nabla \widehat{v} \cdot \nabla \widehat{w}\right) \mathrm{d} \widehat{\Omega} \mathrm{d} \tau \quad \forall \widehat{v}, \widehat{w} \in \widehat{\mathcal{X}}_{h}
$$

$$
\widehat{\mathbf{A}}=\gamma \mathbf{W}_{t} \otimes \widehat{\mathbf{M}}_{s}+\nu \mathbf{M}_{t} \otimes \widehat{\mathbf{K}}_{s},
$$

where $\widehat{\mathbf{K}}_{s}$ and $\widehat{\mathbf{M}}_{s}$ are the equivalent of $(3.10 \mathrm{~b})$ in the parametric domain, i.e. we define for $i, j=1, \ldots, N_{s}$

$$
\left[\widehat{\mathbf{K}}_{s}\right]_{i, j}=\int_{\widehat{\Omega}} \nabla \widehat{B}_{i, p_{s}}(\boldsymbol{\eta}) \cdot \nabla \widehat{B}_{j, p_{s}}(\boldsymbol{\eta}) \mathrm{d} \widehat{\Omega} \quad \text { and } \quad\left[\widehat{\mathbf{M}}_{s}\right]_{i, j}=\int_{\widehat{\Omega}} \widehat{B}_{i, p_{s}}(\boldsymbol{\eta}) \widehat{B}_{j, p_{s}}(\boldsymbol{\eta}) \mathrm{d} \widehat{\Omega} .
$$


We emphasize that the time matrices appearing in 4.1) are the same ones appearing in the system matrix 3.9. This is because for $i, j=1, \ldots, n_{t}$ we have

$$
\left[\mathbf{W}_{t}\right]_{i, j}=\int_{0}^{T} b_{j, p_{t}}^{\prime}(t) b_{i, p_{t}}(t) \mathrm{dt}=\int_{0}^{1} \widehat{b}_{j, p_{t}}^{\prime}(\tau) \widehat{b}_{i, p_{t}}(\tau) \mathrm{d} \tau
$$

and

$$
\left[\mathbf{M}_{t}\right]_{i, j}=\int_{0}^{T} b_{j, p_{t}}(t) b_{i, p_{t}}(t) \mathrm{dt}=T \int_{0}^{1} \widehat{b}_{j, p_{t}}(\tau) \widehat{b}_{i, p_{t}}(\tau) \mathrm{d} \tau .
$$

Thanks to (2.1), the spatial matrices 4.2 have the following structure

$$
\widehat{\mathbf{K}}_{s}=\sum_{l=1}^{d} \widehat{\mathbf{M}}_{d} \otimes \cdots \otimes \widehat{\mathbf{M}}_{l+1} \otimes \widehat{\mathbf{K}}_{l} \otimes \widehat{\mathbf{M}}_{l-1} \otimes \cdots \otimes \widehat{\mathbf{M}}_{1} \quad \text { and } \quad \widehat{\mathbf{M}}_{s}=\widehat{\mathbf{M}}_{d} \otimes \cdots \otimes \widehat{\mathbf{M}}_{1} \text {, }
$$

where for $l=1, \ldots, d$ and for $i, j=1, \ldots, n_{s, l}$ we define

$$
\left[\widehat{\mathbf{K}}_{l}\right]_{i, j}:=\int_{0}^{1} \widehat{b}_{i, p_{s}}^{\prime}\left(\eta_{k}\right) \widehat{b}_{j, p_{s}}^{\prime}\left(\eta_{k}\right) \mathrm{d} \eta_{k} \quad \text { and } \quad\left[\widehat{\mathbf{M}}_{l}\right]_{i, j}:=\int_{0}^{1} \widehat{b}_{i, p_{s}}\left(\eta_{k}\right) \widehat{b}_{j, p_{s}}\left(\eta_{k}\right) \mathrm{d} \eta_{k}
$$

The efficient application of the proposed preconditioner, that is, the solution of a linear system with matrix $\widehat{\mathbf{A}}$, should exploit the structure highlighted above. When the pencils $\left(\mathbf{W}_{t}, \mathbf{M}_{t}\right),\left(\widehat{\mathbf{K}}_{1}, \widehat{\mathbf{M}}_{1}\right), \ldots,\left(\widehat{\mathbf{K}}_{d}, \widehat{\mathbf{M}}_{d}\right)$ admit a stable generalized eigendecomposition, a possible approach is the Fast Diagonalization (FD) method, see 33 and 25 for details. We will see in Section 4.1 that the spatial pencils $\left(\widehat{\mathbf{K}}_{1}, \widehat{\mathbf{M}}_{1}\right), \ldots,\left(\widehat{\mathbf{K}}_{d}, \widehat{\mathbf{M}}_{d}\right)$ admit a stable diagonalization, but this is not the case of $\left(\mathbf{W}_{t}, \mathbf{M}_{t}\right)$, that needs a special treatment as explained in Section 4.2

\subsection{Stable factorization of the pencils $\left(\widehat{\mathbf{K}}_{l}, \widehat{\mathbf{M}}_{l}\right)$ for $l=1, \ldots, d$}

The spatial stiffness and mass matrices $\widehat{\mathbf{K}}_{l}$ and $\widehat{\mathbf{M}}_{l}$ are symmetric and positive definite for $l=1, \ldots, d$. Thus, the pencils $\left(\widehat{\mathbf{K}}_{l}, \widehat{\mathbf{M}}_{l}\right)$ for $l=1, \ldots, d$ admit the generalized eigendecomposition

$$
\widehat{\mathbf{K}}_{l} \mathbf{U}_{l}=\widehat{\mathbf{M}}_{l} \mathbf{U}_{l} \boldsymbol{\Lambda}_{l}
$$

where the matrices $\mathbf{U}_{l}$ contain in each column the $\widehat{\mathbf{M}}_{l}$-orthonormal generalized eigenvectors and $\boldsymbol{\Lambda}_{l}$ are diagonal matrices whose entries contain the generalized eigenvalues. Therefore we have for $l=1, \ldots, d$ the factorizations

$$
\mathbf{U}_{l}^{T} \widehat{\mathbf{K}}_{l} \mathbf{U}_{l}=\boldsymbol{\Lambda}_{l} \quad \text { and } \quad \mathbf{U}_{l}^{T} \widehat{\mathbf{M}}_{l} \mathbf{U}_{l}=\mathbb{I}_{n_{s, l}},
$$

where $\mathbb{I}_{n_{s, l}}$ denotes the identity matrix of dimension $n_{s, l} \times n_{s, l}$. The stability of the decomposition (4.4) is expressed by the condition number of the eigenvector matrix. In particular $\mathbf{U}_{l}^{T} \widehat{\mathbf{M}}_{l} \mathbf{U}_{l}=\mathbb{I}_{n_{s, l}}$ implies that

$$
\kappa_{2}\left(\mathbf{U}_{l}\right):=\left\|\mathbf{U}_{l}\right\|_{2}\left\|\mathbf{U}_{l}^{-1}\right\|_{2}=\sqrt{\kappa_{2}\left(\widehat{\mathbf{M}}_{l}\right)}
$$

where $\|\cdot\|_{2}$ is the norm induced by the Euclidean vector norm. The condition number $\kappa_{2}\left(\widehat{\mathbf{M}}_{l}\right)$ has been studied in 34 and it does not depend on the mesh-size, but it depends on the polynomial degree. Indeed, we report in Table 1 the behavior of $\kappa_{2}\left(\mathbf{U}_{l}\right)$ for different values of spline degree $p_{s}$ and for different uniform discretizations with number of elements denoted by $n_{e l}$. We observe that $\kappa_{2}\left(\mathbf{U}_{l}\right)$ exhibits a dependence only on $p_{s}$, but stays moderately low for all low polynomial degrees that are in the range of interest. 


\begin{tabular}{|r|c|c|c|c|c|c|c|}
\hline$n_{e l}$ & $p_{s}=2$ & $p_{s}=3$ & $p_{s}=4$ & $p_{s}=5$ & $p_{s}=6$ & $p_{s}=7$ & $p_{s}=8$ \\
\hline 32 & $2.7 \cdot 10^{0}$ & $4.5 \cdot 10^{0}$ & $7.6 \cdot 10^{0}$ & $1.3 \cdot 10^{1}$ & $2.1 \cdot 10^{1}$ & $3.5 \cdot 10^{1}$ & $5.7 \cdot 10^{1}$ \\
\hline 64 & $2.7 \cdot 10^{0}$ & $4.5 \cdot 10^{0}$ & $7.6 \cdot 10^{0}$ & $1.3 \cdot 10^{1}$ & $2.1 \cdot 10^{1}$ & $3.5 \cdot 10^{1}$ & $5.7 \cdot 10^{1}$ \\
\hline 128 & $2.7 \cdot 10^{0}$ & $4.5 \cdot 10^{0}$ & $7.6 \cdot 10^{0}$ & $1.3 \cdot 10^{1}$ & $2.1 \cdot 10^{1}$ & $3.5 \cdot 10^{1}$ & $5.7 \cdot 10^{1}$ \\
\hline 256 & $2.7 \cdot 10^{0}$ & $4.5 \cdot 10^{0}$ & $7.6 \cdot 10^{0}$ & $1.3 \cdot 10^{1}$ & $2.1 \cdot 10^{1}$ & $3.5 \cdot 10^{1}$ & $5.7 \cdot 10^{1}$ \\
\hline 512 & $2.7 \cdot 10^{0}$ & $4.5 \cdot 10^{0}$ & $7.6 \cdot 10^{0}$ & $1.3 \cdot 10^{1}$ & $2.1 \cdot 10^{1}$ & $3.5 \cdot 10^{1}$ & $5.7 \cdot 10^{1}$ \\
\hline 1024 & $2.7 \cdot 10^{0}$ & $4.5 \cdot 10^{0}$ & $7.6 \cdot 10^{0}$ & $1.3 \cdot 10^{1}$ & $2.1 \cdot 10^{1}$ & $3.5 \cdot 10^{1}$ & $5.7 \cdot 10^{1}$ \\
\hline
\end{tabular}

TABLE 1. $\kappa_{2}\left(\mathbf{U}_{l}\right)$ for different polynomial degrees $p_{s}$ and number of elements $n_{e l}$.

\subsection{Stable factorization of the pencil $\left(\mathbf{W}_{t}, \mathbf{M}_{t}\right)$}

\subsubsection{Numerical instability of the eigendecomposition}

While $\mathbf{M}_{t}$ is symmetric, $\mathbf{W}_{t}$ is neither symmetric nor skew-symmetric. Indeed

$$
\left[\mathbf{W}_{t}\right]_{i, j}+\left[\mathbf{W}_{t}\right]_{j, i}=\int_{0}^{T} b_{j, p_{t}}^{\prime}(t) b_{i, p_{t}}(t) \mathrm{dt}+\int_{0}^{T} b_{i, p_{t}}^{\prime}(t) b_{j, p_{t}}(t) \mathrm{dt}=b_{i, p_{t}}(T) b_{j, p_{t}}(T)
$$

where $b_{i, p_{t}}(T) b_{j, p_{t}}(T)$ vanishes for all $i=1, \ldots, n_{t}-1$ or $j=1, \ldots, n_{t}-1$. A numerical computation of the generalized eigendecomposition of the pencil $\left(\mathbf{W}_{t}, \mathbf{M}_{t}\right)$, that is

$$
\mathbf{W}_{t} \mathbf{U}=\mathbf{M}_{t} \mathbf{U} \boldsymbol{\Lambda}_{t},
$$

where $\boldsymbol{\Lambda}_{t}$ is the diagonal matrix of the generalized complex eigenvalues and $\mathbf{U}$ is the complex matrix whose columns are the generalized eigenvectors normalized w.r.t. the norm induced by $\mathbf{M}_{t}$, reveals that the eigenvectors are far from $\mathbf{M}_{t}$-orthogonality, i.e. the matrix $\mathbf{U}^{*} \mathbf{M}_{t} \mathbf{U}$ is not diagonal. We set $T=1$ and we report in Table 2 the condition number $\kappa_{2}(\mathbf{U})$ for different values of spline degree $p_{t}$ and for different uniform discretizations with $n_{e l}$ number of elements. In contrast to the spatial case (see Section 4.1), $\kappa_{2}(\mathbf{U}$ ) is large and grows exponentially with respect to the spline degree $p_{t}$ and the level of mesh refinement. This test clearly indicates a numerical instability when computing the generalized eigendecomposition of $\left(\mathbf{W}_{t}, \mathbf{M}_{t}\right)$. A similar behavior has also been highlighted in [15].

\begin{tabular}{|r|c|c|c|c|c|c|c|}
\hline$n_{e l}$ & $p_{t}=2$ & $p_{t}=3$ & $p_{t}=4$ & $p_{t}=5$ & $p_{t}=6$ & $p_{t}=7$ & $p_{t}=8$ \\
\hline 32 & $8.9 \cdot 10^{2}$ & $3.0 \cdot 10^{4}$ & $5.0 \cdot 10^{4}$ & $3.4 \cdot 10^{5}$ & $3.1 \cdot 10^{6}$ & $4.2 \cdot 10^{7}$ & $7.0 \cdot 10^{8}$ \\
\hline 64 & $4.4 \cdot 10^{3}$ & $2.6 \cdot 10^{5}$ & $5.0 \cdot 10^{5}$ & $5.4 \cdot 10^{6}$ & $8.9 \cdot 10^{7}$ & $3.1 \cdot 10^{9}$ & $2.0 \cdot 10^{10}$ \\
\hline 128 & $2.3 \cdot 10^{4}$ & $1.2 \cdot 10^{6}$ & $5.8 \cdot 10^{6}$ & $1.0 \cdot 10^{8}$ & $3.0 \cdot 10^{9}$ & $6.4 \cdot 10^{11}$ & $1.3 \cdot 10^{12}$ \\
\hline 256 & $1.2 \cdot 10^{5}$ & $9.4 \cdot 10^{6}$ & $7.6 \cdot 10^{7}$ & $2.1 \cdot 10^{9}$ & $1.2 \cdot 10^{11}$ & $1.2 \cdot 10^{13}$ & $2.1 \cdot 10^{13}$ \\
\hline 512 & $7.0 \cdot 10^{5}$ & $8.3 \cdot 10^{7}$ & $1.1 \cdot 10^{9}$ & $4.9 \cdot 10^{10}$ & $4.5 \cdot 10^{12}$ & $3.6 \cdot 10^{13}$ & $4.9 \cdot 10^{12}$ \\
\hline 1024 & $4.1 \cdot 10^{6}$ & $8.0 \cdot 10^{8}$ & $1.9 \cdot 10^{10}$ & $1.3 \cdot 10^{12}$ & $9.6 \cdot 10^{12}$ & $1.4 \cdot 10^{12}$ & $5.6 \cdot 10^{12}$ \\
\hline
\end{tabular}

TABLE 2. $\kappa_{2}(\mathbf{U})$ for different degree $p_{t}$ and number of elements $n_{e l}$.

\subsubsection{Construction of the stable factorization}

The analysis above motivates the search of a different but stable factorization of the pencil $\left(\mathbf{W}_{t}, \mathbf{M}_{t}\right)$. We look now for a factorization of the form

$$
\mathbf{W}_{t} \mathbf{U}_{t}=\mathbf{M}_{t} \mathbf{U}_{t} \boldsymbol{\Delta}_{t}
$$

where $\boldsymbol{\Delta}_{t}$ is a complex arrowhead matrix, i.e. with non-zero entries allowed on the diagonal, on the last row and on the last column only. We also require that $\mathbf{U}_{t}$ fulfils the orthogonality condition

$$
\mathbf{U}_{t}^{*} \mathbf{M}_{t} \mathbf{U}_{t}=\mathbb{I}_{n_{t}} .
$$


From 4.7-4.8 we then obtain the factorizations

$$
\mathbf{U}_{t}^{*} \mathbf{W}_{t} \mathbf{U}_{t}=\boldsymbol{\Delta}_{t} \quad \text { and } \quad \mathbf{U}_{t}^{*} \mathbf{M}_{t} \mathbf{U}_{t}=\mathbb{I}_{n_{t}} .
$$

With this aim, we look for $\mathbf{U}_{t}$ as follows:

$$
\mathbf{U}_{t}:=\left[\begin{array}{ll}
\mathbf{U}_{t} & \mathbf{r} \\
\mathbf{0}^{T} & \rho
\end{array}\right]
$$

where $\stackrel{\circ}{\mathbf{U}}_{t} \in \mathbb{C}^{\left(n_{t}-1\right) \times\left(n_{t}-1\right)}, \mathbf{r} \in \mathbb{C}^{n_{t}-1}, \rho \in \mathbb{C}$ and where $\mathbf{0} \in \mathbb{R}^{n_{t}-1}$ denotes the null vector. In order to guarantee the non-singularity of $\mathbf{U}_{t}$, we further impose $\rho \neq 0$. Accordingly, we split the time matrices $\mathbf{W}_{t}$ and $\mathbf{M}_{t}$ as

$$
\mathbf{W}_{t}=\left[\begin{array}{cc}
\stackrel{\circ}{\mathbf{W}}_{t} & \mathbf{w} \\
-\mathbf{w}^{T} & \omega
\end{array}\right] \quad \text { and } \quad \mathbf{M}_{t}=\left[\begin{array}{cc}
\stackrel{\circ}{\mathbf{M}}_{t} & \mathbf{m} \\
\mathbf{m}^{T} & \mu
\end{array}\right]
$$

where we have defined

$$
\begin{gathered}
\omega:=\left[\mathbf{W}_{t}\right]_{n_{t}, n_{t}}, \quad \mu:=\left[\mathbf{M}_{t}\right]_{n_{t}, n_{t}}, \\
{[\mathbf{w}]_{i}=\left[\mathbf{W}_{t}\right]_{i, n_{t}} \text { and }[\mathbf{m}]_{i}=\left[\mathbf{M}_{t}\right]_{i, n_{t}} \text { for } i=1, \ldots, n_{t}-1,} \\
\left.\left[\stackrel{\circ}{\mathbf{W}}_{t}\right]_{i, j}=\left[\mathbf{W}_{t}\right]_{i, j} \text { and } \stackrel{\stackrel{\circ}{\mathbf{M}}}{t}\right]_{i, j}=\left[\mathbf{M}_{t}\right]_{i, j} \text { for } i, j=1, \ldots, n_{t}-1 .
\end{gathered}
$$

Recalling 4.5, we observe that $\stackrel{\circ}{\mathbf{W}}_{t}$ is skew-symmetric and, since $\stackrel{\circ}{\mathbf{M}}_{t}$ is symmetric, we can write the eigendecomposition of the pencils $\left(\stackrel{\circ}{\mathbf{W}}_{t}, \stackrel{\circ}{\mathbf{M}}_{t}\right)$ :

$$
\stackrel{\circ}{\mathbf{W}}_{t} \stackrel{\circ}{\mathbf{U}}_{t}=\stackrel{\circ}{\mathbf{M}}_{t} \stackrel{\circ}{\mathbf{U}}_{t} \stackrel{\circ}{\boldsymbol{\Lambda}}_{t} \quad \text { with } \quad \stackrel{\circ}{\mathbf{U}}_{t}^{*} \stackrel{\circ}{\mathbf{M}}_{t} \stackrel{\circ}{\mathbf{U}}_{t}=\mathbb{I}_{n_{t}-1}
$$

where $\stackrel{\circ}{\mathbf{U}}_{t}$ contains the complex generalized eigenvectors and $\stackrel{\circ}{\Lambda}_{t}$ is the diagonal matrix of the generalized eigenvalues, that are pairs of complex conjugate pure imaginary numbers plus, eventually, the eigenvalue zero. From (4.10-4.11), it follows

$$
\mathbf{U}_{t}^{*} \mathbf{M}_{t} \mathbf{U}_{t}=\left[\begin{array}{cc} 
& \stackrel{\circ}{\mathbb{I}_{n_{t}-1}} \stackrel{\circ}{\mathbf{U}_{t}^{*} \stackrel{\mathbf{M}}{t}_{t} \mathbf{r}+\stackrel{\circ}{\mathbf{U}}_{t}^{*} \mathbf{m} \rho} \\
\stackrel{\circ}{\mathbf{M}}_{t}^{\circ} \stackrel{\circ}{\mathbf{U}}_{t}+\rho^{*} \mathbf{m}^{T} \stackrel{\mathbf{U}}{t} & {\left[\mathbf{r}^{*} \rho^{*}\right] \mathbf{M}_{t}\left[\begin{array}{c}
\mathbf{r} \\
\rho
\end{array}\right]}
\end{array}\right],
$$

where for the top-left block we have used 4.12.

The orthogonality condition in 4.8 holds if and only if $\mathbf{r}$ and $\rho$ fulfil the two conditions:

$$
\left\{\begin{array}{l}
\stackrel{\circ}{\mathbf{U}_{t}^{*}} \stackrel{\circ}{\mathbf{M}_{t}} \mathbf{r}+\stackrel{\circ}{\mathbf{U}_{t}^{*}} \mathbf{m} \rho=\mathbf{0} \\
{\left[\mathbf{r}^{*} \rho^{*}\right] \mathbf{M}_{t}\left[\begin{array}{l}
\mathbf{r} \\
\rho
\end{array}\right]=1}
\end{array}\right.
$$

In order to compute $\mathbf{r}$ and $\rho$, we first find $\mathbf{v} \in \mathbb{C}^{n_{t}-1}$ such that

$$
\stackrel{\circ}{\mathbf{M}_{t}} \mathbf{v}=-\mathbf{m}
$$

then we normalize the vector $\left[\begin{array}{l}\mathbf{v} \\ 1\end{array}\right]$ w.r.t. the $\|\cdot\|_{\mathbf{M}_{t}}$-norm to get

$$
\left[\begin{array}{l}
\mathbf{r} \\
\rho
\end{array}\right]:=\frac{\left[\begin{array}{l}
\mathbf{v} \\
1
\end{array}\right]}{\left(\left[\mathbf{v}^{*} 1\right] \mathbf{M}_{t}\left[\begin{array}{l}
\mathbf{v} \\
1
\end{array}\right]\right)^{\frac{1}{2}}}
$$


that fulfils 4.13a-4.13b. Finally, we get 4.7 by defining

$$
\boldsymbol{\Delta}_{t}:=\mathbf{U}_{t}^{*} \mathbf{W}_{t} \mathbf{U}_{t}=\left[\begin{array}{cc}
\stackrel{\curlywedge}{\Lambda}_{t} & \mathbf{g} \\
-\mathbf{g}^{*} & \sigma
\end{array}\right]
$$

where $\mathbf{g}:=\stackrel{\circ}{\mathbf{U}}_{t}^{*}\left[\stackrel{\circ}{\mathbf{W}}_{t} \mathbf{w}\right]\left[\begin{array}{l}\mathbf{r} \\ \rho\end{array}\right]$ and $\sigma:=\left[\mathbf{r}^{*} \rho^{*}\right] \mathbf{W}_{t}\left[\begin{array}{l}\mathbf{r} \\ \rho\end{array}\right]$. Note that matrix 4.15 has an arrowhead structure.

To assess the stability of the new decomposition 4.9 , we set $T=1$ and we compute the condition number $\kappa_{2}\left(\mathbf{U}_{t}\right)$ for different values of spline degree $p_{t}$ and for various uniform discretizations with number of elements $n_{e l}$. Thanks to $(4.8)$, we have $\kappa_{2}\left(\mathbf{U}_{t}\right)=\sqrt{\kappa_{2}\left(\mathbf{M}_{t}\right)}$. The results, reported in Table 3 show that the condition numbers $\kappa_{2}\left(\mathbf{U}_{t}\right)$ are uniformly bounded w.r.t. the mesh refinement, they grow with respect to the polynomial degree but they are moderately small for all the degrees of interest. We conclude that the factorization 4.9 for the time pencil $\left(\mathbf{W}_{t}, \mathbf{M}_{t}\right)$ is stable.

\begin{tabular}{|r|c|c|c|c|c|c|c|}
\hline$n_{e l}$ & $p_{t}=2$ & $p_{t}=3$ & $p_{t}=4$ & $p_{t}=5$ & $p_{t}=6$ & $p_{t}=7$ & $p_{t}=8$ \\
\hline 32 & $3.2 \cdot 10^{0}$ & $5.2 \cdot 10^{0}$ & $8.3 \cdot 10^{0}$ & $1.3 \cdot 10^{1}$ & $2.2 \cdot 10^{1}$ & $3.6 \cdot 10^{1}$ & $5.9 \cdot 10^{1}$ \\
\hline 64 & $3.3 \cdot 10^{0}$ & $5.2 \cdot 10^{0}$ & $8.3 \cdot 10^{0}$ & $1.3 \cdot 10^{1}$ & $2.2 \cdot 10^{1}$ & $3.6 \cdot 10^{1}$ & $5.9 \cdot 10^{1}$ \\
\hline 128 & $3.3 \cdot 10^{0}$ & $5.2 \cdot 10^{0}$ & $8.3 \cdot 10^{0}$ & $1.3 \cdot 10^{1}$ & $2.2 \cdot 10^{1}$ & $3.6 \cdot 10^{1}$ & $5.9 \cdot 10^{1}$ \\
\hline 256 & $3.3 \cdot 10^{0}$ & $5.2 \cdot 10^{0}$ & $8.3 \cdot 10^{0}$ & $1.3 \cdot 10^{1}$ & $2.2 \cdot 10^{1}$ & $3.6 \cdot 10^{1}$ & $5.9 \cdot 10^{1}$ \\
\hline 512 & $3.3 \cdot 10^{0}$ & $5.2 \cdot 10^{0}$ & $8.3 \cdot 10^{0}$ & $1.3 \cdot 10^{1}$ & $2.2 \cdot 10^{1}$ & $3.6 \cdot 10^{1}$ & $5.9 \cdot 10^{1}$ \\
\hline 1024 & $3.3 \cdot 10^{0}$ & $5.2 \cdot 10^{0}$ & $8.3 \cdot 10^{0}$ & $1.3 \cdot 10^{1}$ & $2.2 \cdot 10^{1}$ & $3.6 \cdot 10^{1}$ & $5.9 \cdot 10^{1}$ \\
\hline
\end{tabular}

TABLE 3. $\kappa_{2}\left(\mathbf{U}_{t}\right)$ for different degrees $p_{t}$ and number of elements $n_{e l}$.

\subsection{Preconditioner application}

The application of the preconditioner involves the solution of the linear system

$$
\widehat{\mathbf{A}} \mathbf{s}=\mathbf{r}
$$

where $\widehat{\mathbf{A}}$ has the structure 4.1). We are able to efficiently solve system 4.16) by extending the FD method. The starting points, that are involved in the setup of the preconditioner, are the following ones:

- for the pencils $\left(\widehat{\mathbf{K}}_{l}, \widehat{\mathbf{M}}_{l}\right)$ for $l=1, \ldots, d$ we have the factorizations 4.4 ;

- for the pencil $\left(\mathbf{W}_{t}, \mathbf{M}_{t}\right)$ we have the factorization 4.9 ).

Then, by defining $\mathbf{U}_{s}:=\mathbf{U}_{d} \otimes \cdots \otimes \mathbf{U}_{1}$ and $\boldsymbol{\Lambda}_{s}:=\sum_{l=1}^{d} \mathbb{I}_{n_{s, d}} \otimes \cdots \otimes \mathbb{I}_{n_{s, l+1}} \otimes \boldsymbol{\Lambda}_{l} \otimes \mathbb{I}_{n_{s, l-1}} \otimes \cdots \otimes \mathbb{I}_{n_{s, 1}}$, we have for the matrix $\widehat{\mathbf{A}}$ the factorization

$$
\widehat{\mathbf{A}}=\left(\mathbf{U}_{t}^{*} \otimes \mathbf{U}_{s}^{T}\right)^{-1}\left(\gamma \boldsymbol{\Delta}_{t} \otimes \mathbb{I}_{N_{s}}+\nu \mathbb{I}_{n_{t}} \otimes \boldsymbol{\Lambda}_{s}\right)\left(\mathbf{U}_{t} \otimes \mathbf{U}_{s}\right)^{-1}
$$

Note that the second factor in 4.17) has the block-arrowhead structure

$$
\gamma \boldsymbol{\Delta}_{t} \otimes \mathbb{I}_{N_{s}}+\nu \mathbb{I}_{n_{t}} \otimes \boldsymbol{\Lambda}_{s}=\left[\begin{array}{cccc}
\mathbf{H}_{1} & & & \mathbf{B}_{1} \\
& \ddots & & \vdots \\
& & \mathbf{H}_{n_{t}-1} & \mathbf{B}_{n_{t}-1} \\
-\mathbf{B}_{1}^{*} & \ldots & -\mathbf{B}_{n_{t}-1}^{*} & \mathbf{H}_{n_{t}}
\end{array}\right]
$$

where $\mathbf{H}_{i}$ and $\mathbf{B}_{i}$ are diagonal matrices defined as

$$
\mathbf{H}_{i}:=\gamma\left[\boldsymbol{\Lambda}_{t}\right]_{i, i} \mathbb{I}_{N_{s}}+\nu \boldsymbol{\Lambda}_{s} \quad \text { and } \quad \mathbf{B}_{i}:=\gamma[\mathbf{g}]_{i} \mathbb{I}_{N_{s}} \quad \text { for } \quad i=1, \ldots, n_{t}-1
$$




$$
\mathbf{H}_{n_{t}}:=\gamma \sigma \mathbb{I}_{N_{s}}+\nu \boldsymbol{\Lambda}_{s} .
$$

The matrix 4.18 has the following easy-to-invert block LU decomposition

$$
\begin{aligned}
\gamma \boldsymbol{\Delta}_{t} \otimes \mathbb{I}_{N_{s}}+\nu \mathbb{I}_{n_{t}} \otimes \boldsymbol{\Lambda}_{s} & \\
& =\left[\begin{array}{ccccc}
\mathbb{I}_{N_{s}} & & & \\
& \ddots & & \\
& & \mathbb{I}_{N_{s}} & \\
-\mathbf{B}_{1}^{*} \mathbf{H}_{1}^{-1} & \ldots & -\mathbf{B}_{n_{t}-1}^{*} \mathbf{H}_{n_{t}-1}^{-1} & \mathbb{I}_{N_{s}}
\end{array}\right]\left[\begin{array}{llll}
\mathbf{H}_{1} & & & \mathbf{B}_{1} \\
& \ddots & & \vdots \\
& & \mathbf{H}_{n_{t}-1} & \mathbf{B}_{n_{t}-1} \\
& & & \mathbf{S}
\end{array}\right]
\end{aligned}
$$

where $\mathbf{S}:=\mathbf{H}_{n_{t}}+\sum_{i=1}^{n_{t}-1} \mathbf{B}_{i}^{*} \mathbf{H}_{i}^{-1} \mathbf{B}_{i}$ is a diagonal matrix.

Summarising, the solution of (4.16) can be computed by the following algorithm.

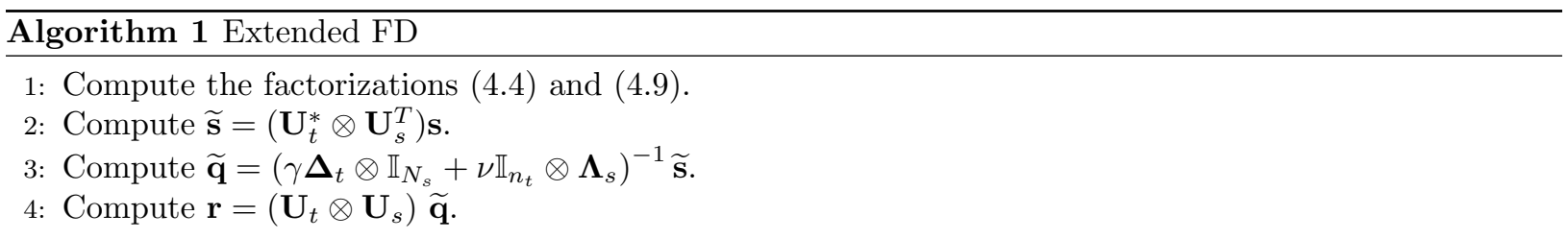

\subsection{Preconditioner robustness: partial inclusion of the geometry}

The preconditioner (4.1) does not incorporate any information on the spatial parametrization $\boldsymbol{F}$. Thus, the quality of the preconditioning strategy may depend on the geometry map: we see this trend in the numerical tests presented in the upper tables of Table 4 and Table 6 of Section 5 . However, we can generalize (4.1) by including in the univariate spatial matrices $\widehat{\mathbf{K}}_{l}, \widehat{\mathbf{M}}_{l}$ for $l=1, \ldots, d$ a suitable approximation of $\boldsymbol{F}$, without increasing the asymptotic computational cost. A similar approach has been used also in 35] for the Stokes problem and in 21 for a least-squares formulation of the heat equation. We briefly give an overview of this strategy.

Referring to Section 2.2 for the notation of the basis functions, we rewrite the entries of the system matrix 3.8 in the parametric domain as

$$
\begin{aligned}
{[\mathbf{A}]_{i, j} } & =\mathcal{A}\left(B_{j, \boldsymbol{p}}, B_{i, \boldsymbol{p}}\right) \\
& =\gamma \int_{0}^{1} \int_{\widehat{\Omega}} \frac{1}{T} \partial_{\tau} \widehat{B}_{j, \boldsymbol{p}} \widehat{B}_{i, \boldsymbol{p}}\left|\operatorname{det}\left(J_{\boldsymbol{G}}\right)\right| \mathrm{d} \widehat{\Omega} \mathrm{d} \tau+\int_{0}^{1} \int_{\widehat{\Omega}} \nu\left(\nabla \widehat{B}_{j, \boldsymbol{p}}\right)^{T} J_{\boldsymbol{G}}^{-1} J_{\boldsymbol{G}}^{-T} \nabla \widehat{B}_{i, \boldsymbol{p}}\left|\operatorname{det}\left(J_{\boldsymbol{G}}\right)\right| \mathrm{d} \widehat{\Omega} \mathrm{d} \tau \\
& =\int_{0}^{1} \int_{\widehat{\Omega}}\left[\left(\nabla \widehat{B}_{j, \boldsymbol{p}}\right)^{T} \partial_{\tau} \widehat{B}_{j, \boldsymbol{p}}\right]\left[\begin{array}{ll}
\nu T \mathbb{I}_{d} & \\
& \gamma
\end{array}\right] \mathfrak{C}\left[\begin{array}{ll}
\left(\nabla \widehat{B}_{i, \boldsymbol{p}}\right)^{T} & \widehat{B}_{i, \boldsymbol{p}}
\end{array}\right]^{T} \mathrm{~d} \widehat{\Omega} \mathrm{d} \tau,
\end{aligned}
$$

where

$$
\mathfrak{C}:=\left[\begin{array}{ll}
J_{\boldsymbol{F}}^{-1} J_{\boldsymbol{F}}^{-T}\left|\operatorname{det}\left(J_{\boldsymbol{F}}\right)\right| & \\
& \left|\operatorname{det}\left(J_{\boldsymbol{F}}\right)\right|
\end{array}\right]
$$

and where we used that $B_{i, \boldsymbol{p}}=\widehat{B}_{i, \boldsymbol{p}} \circ \boldsymbol{G}^{-1}, B_{j, \boldsymbol{p}}=\widehat{B}_{j, \boldsymbol{p}} \circ \boldsymbol{G}^{-1}$ and $\left|\operatorname{det}\left(J_{\boldsymbol{G}}\right)\right|=T\left|\operatorname{det}\left(J_{\boldsymbol{F}}\right)\right|$. The construction of the preconditioner is based on the following approximation of the diagonal entries only of $\mathfrak{C}$ :

$$
\begin{aligned}
& {[\mathfrak{C}(\boldsymbol{\eta})]_{l, l} \approx[\widetilde{\mathfrak{C}}(\boldsymbol{\eta})]_{l, l}:=\varphi_{1}\left(\eta_{1}\right) \ldots \varphi_{l-1}\left(\eta_{l-1}\right) \Phi_{l}\left(\eta_{l}\right) \varphi_{l+1}\left(\eta_{l+1}\right) \ldots \varphi_{d}\left(\eta_{d}\right) \quad \text { for } l=1, \ldots, d,} \\
& {[\mathfrak{C}(\boldsymbol{\eta})]_{d+1, d+1} \approx[\widetilde{\mathfrak{C}}(\boldsymbol{\eta})]_{d+1, d+1}:=\varphi_{1}\left(\eta_{1}\right) \ldots \varphi_{d}\left(\eta_{d}\right) .}
\end{aligned}
$$

In order to compute such an approximation, we interpolate the functions $[\widetilde{\mathfrak{C}}(\boldsymbol{\eta})]_{l, l}$ in $[4.20$ by piecewise constants in each element and we build the univariate factors $\varphi_{l}$ and $\Phi_{l}$ by using the separation of variables algorithm detailed in [21, Appendix C]. The computational cost of the approximation above is proportional 
to the number of elements in $\Omega$, that, when using smooth B-splines, is almost equal to $N_{s}$, independent of $p_{s}$ and $p_{t}$ and thus negligible in the whole iterative strategy.

Then we define

$$
[\widetilde{\mathbf{A}}]_{i, j}:=\int_{0}^{1} \int_{\widehat{\Omega}}\left[\left(\nabla \widehat{B}_{j, \boldsymbol{p}}\right)^{T} \partial_{\tau} \widehat{B}_{j, \boldsymbol{p}}\right]\left[\begin{array}{ll}
\nu T \mathbb{I}_{d} & \\
& \gamma
\end{array}\right] \widetilde{\mathfrak{C}}\left[\begin{array}{ll}
\left(\nabla \widehat{B}_{i, \boldsymbol{p}}\right)^{T} & \widehat{B}_{i, \boldsymbol{p}}
\end{array}\right]^{T} \mathrm{~d} \widehat{\Omega} \mathrm{d} \tau .
$$

The previous matrix maintains the same Kronecker structure as 4.1). Indeed we have that

$$
\widetilde{\mathbf{A}}=\gamma \mathbf{W}_{t} \otimes \widetilde{\mathbf{M}}_{s}+\nu \mathbf{M}_{t} \otimes \widetilde{\mathbf{K}}_{s}
$$

where

$$
\widetilde{\mathbf{K}}_{s}:=\sum_{l=1}^{d} \widetilde{\mathbf{M}}_{d} \otimes \cdots \otimes \widetilde{\mathbf{M}}_{l+1} \otimes \widetilde{\mathbf{K}}_{l} \otimes \widetilde{\mathbf{M}}_{l-1} \otimes \cdots \otimes \widetilde{\mathbf{M}}_{1}, \quad \widetilde{\mathbf{M}}_{s}:=\widetilde{\mathbf{M}}_{d} \otimes \cdots \otimes \widetilde{\mathbf{M}}_{1}
$$

and where for $l=1, \ldots, d$ and for $i, j=1, \ldots, n_{s, l}$ we define

$$
\left[\widetilde{\mathbf{K}}_{l}\right]_{i, j}:=\int_{0}^{1} \Phi_{l}\left(\eta_{l}\right) \widehat{b}_{i, p_{s}}^{\prime}\left(\eta_{l}\right) \widehat{b}_{j, p_{s}}^{\prime}\left(\eta_{l}\right) \mathrm{d} \eta_{l} \text { and }\left[\widetilde{\mathbf{M}}_{l}\right]_{i, j}:=\int_{0}^{1} \varphi_{l}\left(\eta_{l}\right) \widehat{b}_{i, p_{s}}\left(\eta_{l}\right) \widehat{b}_{j, p_{s}}\left(\eta_{l}\right) \mathrm{d} \eta_{l} \text {. }
$$

We remark that the application of 4.21 can still be performed by Algorithm 1. Finally, we apply a diagonal scaling on $\widetilde{\mathbf{A}}$ and we define the preconditioner as

$$
\widehat{\mathbf{A}}^{G}:=\mathbf{D}^{\frac{1}{2}} \widetilde{\mathbf{A}} \mathbf{D}^{\frac{1}{2}}
$$

where $[\mathbf{D}]_{i, i}:=\frac{[\mathbf{A}]_{i, i}}{[\widetilde{\mathbf{A}}]_{i, i}}$ for $i=1, \ldots, N_{d o f}$.

\subsubsection{The case of non-constant separable coefficients}

We briefly discuss a generalization of the preconditioning strategy to the case of non-constant equation coefficients $\gamma$ and $\nu$. We assume that $\gamma$ and $\nu$ are positive functions defined over $\Omega \times[0, T]$ and that they are separable in space and in time, i.e. we can write

$$
\gamma(\boldsymbol{x}, t)=\gamma_{s}(\boldsymbol{x}) \gamma_{t}(t), \quad \nu(\boldsymbol{x}, t)=\nu_{s}(\boldsymbol{x}) \nu_{t}(t),
$$

with $\gamma_{s}, \gamma_{t}, \nu_{s}$ and $\nu_{t}$ positive functions.

Now, the first equation of 3.1 can be written as

$$
\gamma_{s} \partial_{t} u-\nabla \cdot\left(\frac{\nu_{t}}{\gamma_{t}} \nu_{s} \nabla u\right)=\frac{f}{\gamma_{t}}
$$

We discretize this equation as described in Section 3 and we generalize the definition of the linear system 3.9 with

$$
\mathbf{A}:=\mathbf{W}_{t} \otimes \underline{\mathbf{M}}_{s}+\underline{\mathbf{M}}_{t} \otimes \underline{\mathbf{K}}_{s}
$$

where $\mathbf{W}_{t}$ is defined as in 3.10a, while for $i, j=1, \ldots, n_{t}$

$$
\left[\underline{\mathbf{M}}_{t}\right]_{i, j}:=\int_{0}^{T} \frac{\nu_{t}(t)}{\gamma_{t}(t)} b_{i, p_{t}}(t) b_{j, p_{t}}(t) \mathrm{dt}
$$

and for $i, j=1, \ldots, N_{s}$

$$
\left[\underline{\mathbf{M}}_{s}\right]_{i, j}:=\int_{\Omega} \gamma_{s}(\boldsymbol{x}) B_{i, \boldsymbol{p}_{s}}(\boldsymbol{x}) B_{j, \boldsymbol{p}_{s}}(\boldsymbol{x}) \mathrm{d} \Omega \text { and }\left[\underline{\mathbf{K}}_{s}\right]_{i, j}:=\int_{\Omega} \nu_{s}(\boldsymbol{x}) \nabla B_{i, \boldsymbol{p}_{s}}(\boldsymbol{x}) \cdot \nabla B_{j, \boldsymbol{p}_{s}}(\boldsymbol{x}) \mathrm{d} \Omega
$$


Then, the preconditioner that we propose is defined as in 4.22

$$
\widehat{\mathbf{A}}^{G}:=\mathbf{D}^{\frac{1}{2}} \widetilde{\mathbf{A}} \mathbf{D}^{\frac{1}{2}}
$$

but here we generalize 4.21 with

$$
\widetilde{\mathbf{A}}:=\mathbf{W}_{t} \otimes \breve{\mathbf{M}}_{s}+\underline{\mathbf{M}}_{t} \otimes \breve{\mathbf{K}}_{s}
$$

where the matrices $\breve{\mathbf{K}}_{s}$ and $\breve{\mathbf{M}}_{s}$ are obtained by using an approximation technique analogous to the one described previously in this section, with $\gamma_{s}$ and $\nu_{s}$ included in the coefficient matrix $\mathfrak{C}$. The preconditioner $\widetilde{\mathbf{A}}$ can still be applied as described in Section 4.3. Note that, for this purpose, it is crucial that $\mathbf{W}_{t}$ does not incorporate any time-dependent coefficient, since this would invalidate (4.5).

\subsection{Computational cost and memory requirement}

The matrix $(3.9)$ is neither positive definite nor symmetric and we choose GMRES as linear solver for the system (3.8). In GMRES, the orthogonalization of the basis of the Krylov subspace makes the computational cost nonlinear with respect to the number of iterations. However, as long as this number is not too high, at each iteration the two dominant costs are the application of the preconditioning strategy and the computation of the residual.

We assume, for simplicity that for $l=1, \ldots, d$ the matrices $\widehat{\mathbf{K}}_{l}, \widehat{\mathbf{M}}_{l}$ and $\widetilde{\mathbf{K}}_{l}, \widetilde{\mathbf{M}}_{l}$ have dimensions $n_{s} \times n_{s}$ and that the matrices $\mathbf{W}_{t}, \mathbf{M}_{t}$ and $\widetilde{\mathbf{W}}_{t}, \widetilde{\mathbf{M}}_{t}$ have dimensions $n_{t} \times n_{t}$. Thus the total number of degrees-offreedom is $N_{\text {dof }}=N_{s} n_{t}=n_{s}^{d} n_{t}$.

The setup of $\widehat{\mathbf{A}}$ and $\widehat{\mathbf{A}}^{\boldsymbol{G}}$ includes the operations performed in Step 1 of Algorithm 1, i.e. $d$ spatial eigendecompositions, that have a total cost of $O\left(d n_{s}^{3}\right)$ FLOPs, and the factorization of the time matrices. The computational cost of the latter, that is the sum of the cost of the eigendecomposition (4.12) and of the cost to compute the solution $\mathbf{v}$ of the linear system (4.14), yields a cost of $O\left(n_{t}^{3}\right)$ FLOPs. Then, the total cost of the spatial and time factorizations is $O\left(d n_{s}^{3}+n_{t}^{3}\right)$ FLOPs. Note that, if $n_{t}=O\left(n_{s}\right)$, this cost is optimal for $d=2$ and negligible for $d=3$. The setup cost of $\widehat{\mathbf{A}}^{G}$ includes also the the construction of the diagonal matrix $\mathbf{D}$, that has a negligible cost, and the computation of the $2 d$ approximations $\varphi_{1}, \ldots, \varphi_{d}$ and $\Phi_{1}, \ldots, \Phi_{d}$ in 4.20), whose cost is negligible too, as mentioned in Section 4.4. We remark that the setup of the preconditioners has to be performed only once, since the matrices involved do not change during the iterative procedure.

The application of the preconditioner is performed by Steps 2-4 of Algorithm 1. Exploiting (2.5), Step 2 and Step $4 \operatorname{costs} 4\left(d n_{s}^{d+1} n_{t}+n_{t}^{2} n_{s}^{d}\right)=4 N_{d o f}\left(d n_{s}+n_{t}\right)$ FLOPs. The use of the block LU decomposition (4.19) makes the cost for Step 3 equal to $O\left(N_{d o f}\right)$ FLOPs.

In conclusion, the total cost of Algorithm 1 is $4 N_{d o f}\left(d n_{s}+n_{t}\right)+O\left(N_{d o f}\right)$ FLOPs. The non-optimal dominant cost of Step 2 and Step 4 is determined by the dense matrix-matrix products. However, these operations are usually implemented on modern computers in a very efficient way. For this reason, in our numerical tests, the overall serial computational time grows almost as $O\left(N_{d o f}\right)$, see Figure 3 in Section 5 .

The other dominant computational cost in a GMRES iteration is the cost of the residual computation, that is the multiplication of the matrix $\mathbf{A}$ with a vector. This multiplication is done by exploiting the special structure (3.9), that allows a matrix-free approach and the use of formula 2.5. Note in particular that we do not need to compute and to store the whole matrix $\mathbf{A}$, but only its time and spatial factors. Since the time matrices $\mathbf{M}_{t}$ and $\mathbf{W}_{t}$ are banded with a band of width $2 p_{t}+1$ and the spatial matrices $\mathbf{K}_{s}$ and $\mathbf{M}_{s}$ have roughly $N_{s}\left(2 p_{s}+1\right)^{d}$ nonzero entries, we have that the computational cost of a single matrix-vector product is $6 N_{d o f}\left[\left(2 p_{s}+1\right)^{d}+2 p_{t}+1\right] \approx 6 N_{d o f}(2 p+1)^{d}=O\left(N_{d o f} p^{d}\right)$ FLOPs, if we assume $p=p_{s} \approx p_{t}$. The numerical experiments reported in Table 5 of Section 5 show that the dominant cost in the iterative solver is represented by the residual computation. This is a typical behaviour of the FD-based preconditioning strategies, see [21, 35, 22].

We now investigate the memory consumption. For the preconditioner we have to store the eigenvector spatial matrices $\mathbf{U}_{1}, \ldots, \mathbf{U}_{d}$, the time matrix $\mathbf{U}_{t}$ and the block-arrowhead matrix 4.18. The memory required is roughly

$$
n_{t}^{2}+d n_{s}^{2}+2 N_{d o f}
$$


For the system matrix, we have to store the time factors $\mathbf{M}_{t}$ and $\mathbf{W}_{t}$ and the spatial factors $\mathbf{M}_{s}$ and $\mathbf{K}_{s}$. Thus the memory required is roughly

$$
2\left(2 p_{t}+1\right) n_{t}+2\left(2 p_{s}+1\right)^{d} N_{s} \approx 4 p_{t} n_{t}+2^{d+1} p_{s}^{d} N_{s} .
$$

Analogously to the least-squares case of [21, we conclude that, in terms of memory requirement, our approach is very attractive w.r.t. other approaches, e.g. the ones obtained by discretizing in space and in time separately. For example if we assume $d=3, p_{t} \approx p_{s}=p$ and $n_{t}^{2} \leq C p^{3} N_{s}$, then the total memory consumption is $O\left(p^{3} N_{s}+N_{d o f}\right)$, that is equal to the sum of the memory needed to store the Galerkin matrices associated to spatial variables and the memory needed to store the solution of the problem.

We remark that we could avoid storing the factors of $\mathbf{A}$ by using the matrix-free approach of [5]. The memory and the computational cost of the iterative solver would significantly improve, both for the setup and the matrix-vector multiplications. However, we do not pursue this strategy, as it is beyond the scope of this paper.

Remark 2. For a better computational efficiency, we use a real-arithmetic version of Algorithm 1: we replace $\widetilde{\boldsymbol{\Lambda}_{t}}$ in 4.15 by a block diagonal matrix where each pair of generalized eigenvalues $i \lambda_{j}$ and $-i \lambda_{j} i s$ replaced by a diagonal block

$$
\left[\begin{array}{cc}
0 & \lambda_{j} \\
-\lambda_{j} & 0
\end{array}\right]
$$

and we set

$$
\mathbf{H}_{j}:=\left[\begin{array}{cc}
\nu \boldsymbol{\Lambda}_{s} & \gamma \lambda_{j} \mathbb{I}_{n_{s}} \\
-\gamma \lambda_{j} \mathbb{I}_{n_{s}} & \nu \boldsymbol{\Lambda}_{s}
\end{array}\right] \quad \text { and } \quad \mathbf{B}_{j}:=\gamma\left[[\mathbf{g}]_{2(j-1)+1} \mathbb{I}_{N_{s}}, \quad[\mathbf{g}]_{2(j-1)+2} \mathbb{I}_{N_{s}}\right]^{T} .
$$

Note that the computational cost of Step 3 in Algorithm 1 does not change, as we have

$$
\mathbf{H}_{j}^{-1}:=\left[\begin{array}{cc}
\frac{1}{\nu} \boldsymbol{\Lambda}_{s}^{-1}-\frac{\gamma^{2}}{\nu^{2}} \lambda_{j}^{2} \boldsymbol{\Lambda}_{s}^{-1} \mathbf{R}_{j}^{-1} \boldsymbol{\Lambda}_{s}^{-1} & -\frac{\gamma}{\nu} \lambda_{j} \boldsymbol{\Lambda}_{s}^{-1} \mathbf{R}_{j}^{-1} \\
\frac{\gamma}{\nu} \lambda_{j} \mathbf{R}_{j}^{-1} \boldsymbol{\Lambda}_{s}^{-1} & \mathbf{R}_{j}^{-1}
\end{array}\right] .
$$

where $\mathbf{R}_{j}:=\nu \boldsymbol{\Lambda}_{s}+\frac{\gamma^{2}}{\nu} \lambda_{j}^{2} \boldsymbol{\Lambda}_{s}^{-1}$

\section{Numerical Results}

In this section we first present the numerical experiments that assess the convergence behavior of the Galerkin approximation and then we analyze the performance of the preconditioners. We also present a comparison with the the least-squares solver of [21].

We consider only sequential executions and we force the use of a single computational thread in a Intel Core i7-5820K processor, running at $3.30 \mathrm{GHz}$ and with $64 \mathrm{~GB}$ of RAM.

The tests are performed with Matlab R2015a and GeoPDEs toolbox [36. We use the eig Matlab function to compute the generalized eigendecompositions present in Step 1 of Algorithm 1, while Tensorlab toolbox 37] is employed to perform the multiplications with Kronecker matrices occurring in Step 2 and Step 4. The solution of the linear system (4.14) is performed by Matlab direct solver (backslash operator "Y"). The linear system is solved by GMRES, with tolerance equal to $10^{-8}$ and with the null vector as initial guess in all tests. We remark that GMRES computes and stores a full orthonormal basis for the Krylov space, and this might be unfeasible if the number of iterations is too large. This issue could be addressed by switching to a different solver for nonsymmetric systems, like e.g. BiCGStab, or using the restarted version of GMRES.

According to Remark 1 we use the same mesh-size in space and in time $h_{s}=h_{t}=: h$, and use splines of maximal continuity and same degree in space and in time $p_{t}=p_{s}=: p$. For the sake of simplicity, we also consider uniform knot vectors, and denote the number of elements in each parametric direction by $n_{e l}:=\frac{1}{h}$.

In out tables, the symbol "*" denotes that the construction of the matrix factors of $\mathbf{A}$ (see (3.9)) goes out of memory, while the symbol "**" indicates that the dimension of the Krylov subspace is too high and there is not enough memory to store all the GMRES iterates. We remark that in all the tables the total solving time of the iterative strategies includes also the setup time of the considered preconditioner. 


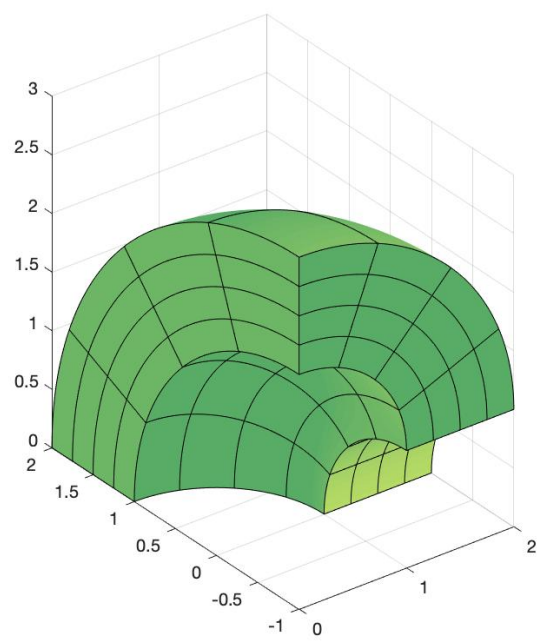

(A) Rotated quarter of annulus.

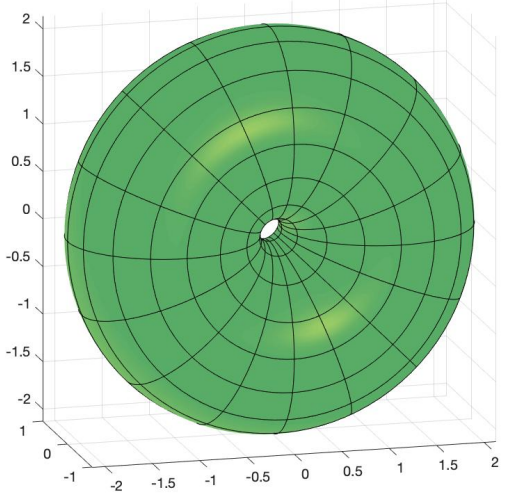

(B) Hollow torus.

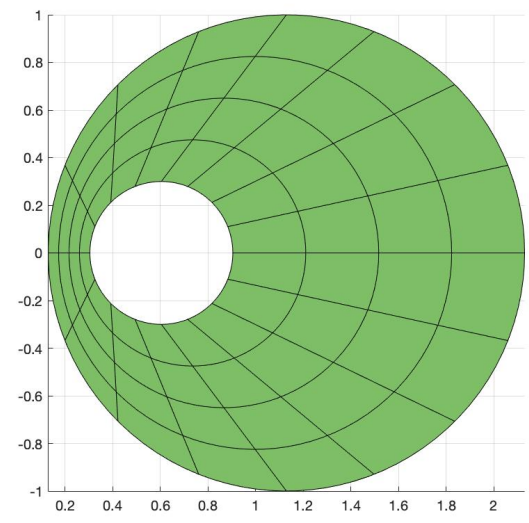

(C) Section of the hollow torus.

Figure 1. Computational domains. 


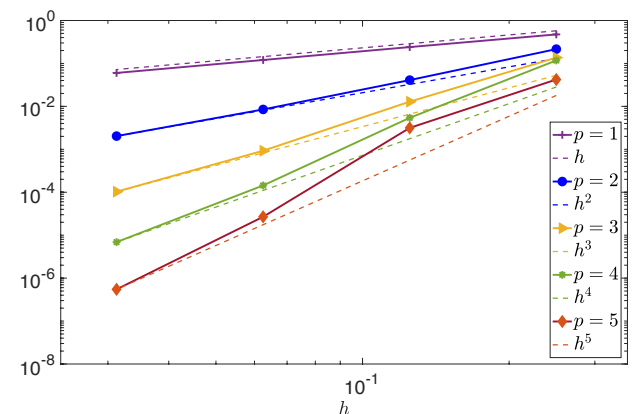

(A) $L^{2}\left(0, T ; H_{0}^{1}(\Omega)\right) \cap H^{1}\left(0, T ; L^{2}(\Omega)\right)$ norm relative errors.

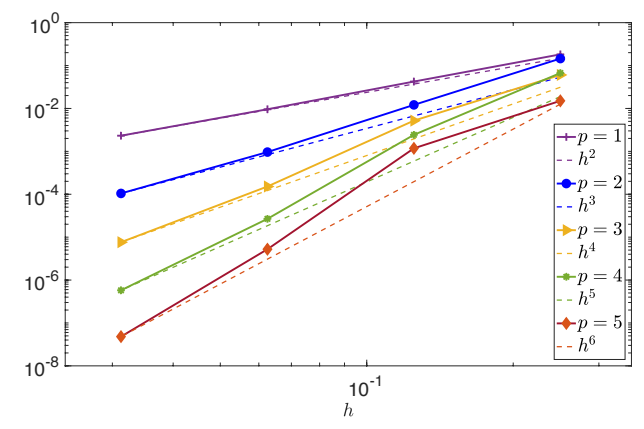

(в) $L^{2}\left(0, T ; L^{2}(\Omega)\right)$ norm relative errors.

Figure 2. Relative errors.

\subsection{Orders of convergence}

We consider as spatial computational domain $\Omega$ a rotated quarter of annulus, represented in Figure 1 a we rotate by $\frac{\pi}{2}$ a quarter of annulus with center in the origin, internal radius 1 and external radius 2 along the axis $\left\{\left(x_{1},-1,0\right) \mid x_{1} \in \mathbb{R}\right\}$. Dirichlet and initial boundary conditions are set such that $u(\boldsymbol{x}, t)=$ $-\left(x_{1}^{2}+x_{2}^{2}-1\right)\left(x_{1}^{2}+x_{2}^{2}-4\right) x_{1} x_{2}^{2} \sin (t) \sin \left(x_{3}\right)$ is the exact solution with constants $\nu=\gamma=1$.

In Figure 2a we represent the relative errors in $L^{2}\left(0, T ; H_{0}^{1}(\Omega)\right) \cap H^{1}\left(0, T ; L^{2}(\Omega)\right)$ norm, an easily computable upper bound of $\|\cdot\|_{\mathcal{X}_{h}}$, for polynomial degrees $p=1,2,3,4,5$. The rates of convergence are optimal, i.e. of order $O\left(h^{p}\right)$, consistent with the a-priori estimate (3.4). Even if this case is not covered by theoretical results, we also compute the relative errors in $L^{2}\left(0, T ; L^{2}(\Omega)\right)$ norm: the orders of convergence are still optimal, that is of order $O\left(h^{p+1}\right)$, as Figure $2 \mathrm{~b}$ shows.

\subsection{Performance of the preconditioner: rotated quarter of annulus}

We consider again as spatial computational domain $\Omega$ the rotated quarter of annulus of Figure 1 a and the same exact solution, initial and boundary data as in Section 5.1 . We analyze the performance of both $\widehat{\mathbf{A}}$ and $\widehat{\mathbf{A}}^{G}$. The maximum dimension of the Krylov subspace is set equal to 100 for both the preconditioners up to $n_{e l}=64$. We are able to reach convergence and to perform the tests with $\widehat{\mathbf{A}}^{\boldsymbol{G}}, n_{e l}=128$ and $p=1,2,3$ by setting the maximum Krylov subspace dimension equal to 25 . In Table 4 we report the number of iterations and the total solving time of GMRES preconditioned with $\widehat{\mathbf{A}}$ (upper table) and $\widehat{\mathbf{A}}^{\boldsymbol{G}}$ (middle table). The non-trivial geometry clearly affects the performance of $\widehat{\mathbf{A}}$, but, when we include some information on the parametrization by using $\widehat{\mathbf{A}}^{\boldsymbol{G}}$, the number of iterations is more than halved and it is stable w.r.t. $p$ and $n_{e l}$. Moreover, the computational times are one order of magnitude lower for the highest degrees and $n_{e l}$. In the lower table of Table 4 we report the results of [21, Section 5, central table of Table 2] obtained by solving the same problem with the least-squares formulation and the related preconditioning strategy. In this case the iterative solver is the preconditioned conjugate gradient method, the tolerance is $10^{-8}$ and the initial guess is the null vector. The number of iterations is more than doubled and the computational times are three times higher than the number of iterations and computational times of $\widehat{\mathbf{A}}^{\boldsymbol{G}}$, in the present setting.

Finally, we analyze with more details the performance of $\widehat{\mathbf{A}}^{G}$. First, we consider the percentage of time spent in the application of $\widehat{\mathbf{A}}^{G}$ in one GMRES iteration. The results, reported in Table 5 , clearly show that the dominant cost consists of the matrix-vector multiplications, while the application of the preconditioner takes a small percentage of the total computational time, for example less than $10 \%$ for polynomial degree 5 and $n_{e l}=32$ or $n_{e l}=64$. In Figure 3 we report the setup time and the single application time of $\widehat{\mathbf{A}}^{\boldsymbol{G}}$ w.r.t. the number of degrees of freedom. As expected, the setup time is proportional to $O\left(N_{\text {dof }}\right)$. What is more interesting is that the application time grows slower than $O\left(N_{d o f}^{5 / 4}\right)$, i.e. the FLOPS counting, and it is almost proportional to $O\left(N_{d o f}\right)$ : this may be explained by the fact that the memory access is the dominant cost due to the high-efficiency of CPU operations, in our case implemented in Matlab Tensorlab [37. 


\begin{tabular}{|c|c|c|c|c|c|}
\hline & \multicolumn{5}{|c|}{$\widehat{\mathbf{A}}$ Iterations / Time } \\
\hline$n_{e l}$ & $p=1$ & $p=2$ & $p=3$ & $p=4$ & $p=5$ \\
\hline 8 & $34 / \quad 0.20$ & $37 / \quad 0.21$ & $42 /$ & $46 /$ & $50 / \quad 1.13$ \\
\hline 16 & 43 / 1.15 & $46 / 1.65$ & $50 /$ & $54 /$ & $57 / \quad 11.87$ \\
\hline 32 & $50 / 22.75$ & $53 / 31.10$ & $57 / \quad 54.02$ & $61 / 96.06$ & $64 / 184.84$ \\
\hline 64 & $57 / 586.73$ & $60 / 764.26$ & $67 / 1254.81$ & $67 / 1858.55$ & $71 / 3188.51$ \\
\hline 128 & $* *$ & ** & ** & * & * \\
\hline
\end{tabular}

\begin{tabular}{|c|c|c|c|c|c|c|c|c|}
\hline & \multicolumn{8}{|c|}{$\widehat{\mathbf{A}}^{G} \quad$ Iterations / Time } \\
\hline$n_{e l}$ & \multicolumn{2}{|c|}{$p=1$} & \multicolumn{2}{|c|}{$p=2$} & \multicolumn{2}{|c|}{$p=3$} & $p=4$ & $p=5$ \\
\hline 8 & $11 /$ & 0.06 & $12 /$ & 0.09 & $12 /$ & 0.11 & $13 / 0.18$ & $14 / \quad 0.29$ \\
\hline 16 & $13 /$ & 0.26 & $14 /$ & 0.52 & $14 /$ & 1.18 & $14 / 1.44$ & $15 / 3.85$ \\
\hline 32 & $15 /$ & 4.73 & $15 /$ & 6.76 & $15 /$ & 12.67 & $15 / 21.47$ & $16 / 40.54$ \\
\hline 64 & $16 /$ & 107.24 & $16 /$ & 135.74 & $18 /$ & 249.27 & $16 / 370.31$ & $17 / 695.44$ \\
\hline 128 & $17 /$ & 23.57 & $17 /$ & 105.76 & $17 /$ & 614.10 & * & * \\
\hline
\end{tabular}

\begin{tabular}{|c|c|c|c|c|c|c|c|}
\hline \multirow{3}{*}{$\frac{n_{e l}}{8}$} & \multicolumn{7}{|c|}{ Least-squares Iterations / Time } \\
\hline & \multicolumn{2}{|c|}{$p_{t}=2$} & \multicolumn{2}{|c|}{$p_{t}=3$} & \multicolumn{2}{|c|}{$p_{t}=4$} & $p_{t}=5$ \\
\hline & $24 /$ & 0.09 & $24 /$ & 0.13 & $26 /$ & 0.37 & $26 /$ \\
\hline 16 & $35 /$ & 0.77 & $34 /$ & 1.96 & $33 /$ & 4.62 & $33 /$ \\
\hline 32 & $42 /$ & 17.03 & $41 /$ & 39.57 & $40 /$ & 82.35 & $41 / 161.73$ \\
\hline 64 & $46 /$ & 333.20 & $44 /$ & 716.03 & $49 /$ & 577.55 & $53 / 3384.08$ \\
\hline 128 & $48 /$ & 767.08 & $50 /$ & 4814.09 & & * & * \\
\hline
\end{tabular}

TABlE 4. Revolved quarter domain. Performance of $\widehat{\mathbf{A}}, \widehat{\mathbf{A}}^{\boldsymbol{G}}$ and the least-squares solver.

\begin{tabular}{|r|c|c|c|c|c|}
\hline$n_{e l}$ & $p=1$ & $p=2$ & $p=3$ & $p=4$ & $p=5$ \\
\hline 8 & $73.02 \%$ & $79.24 \%$ & $66.62 \%$ & $46.94 \%$ & $33.73 \%$ \\
\hline 16 & $68.10 \%$ & $46.13 \%$ & $30.06 \%$ & $17.63 \%$ & $11.27 \%$ \\
\hline 32 & $53.09 \%$ & $33.34 \%$ & $20.44 \%$ & $13.06 \%$ & $8.19 \%$ \\
\hline 64 & $54.71 \%$ & $32.46 \%$ & $20.20 \%$ & $12.52 \%$ & $7.31 \%$ \\
\hline 128 & $54.12 \%$ & $33.53 \%$ & $18.89 \%$ & $*$ & $*$ \\
\hline
\end{tabular}

TABle 5. Percentage of computing time of $\widehat{\mathbf{A}}^{G}$ in one GMRES iteration for the rotated quarter domain. 


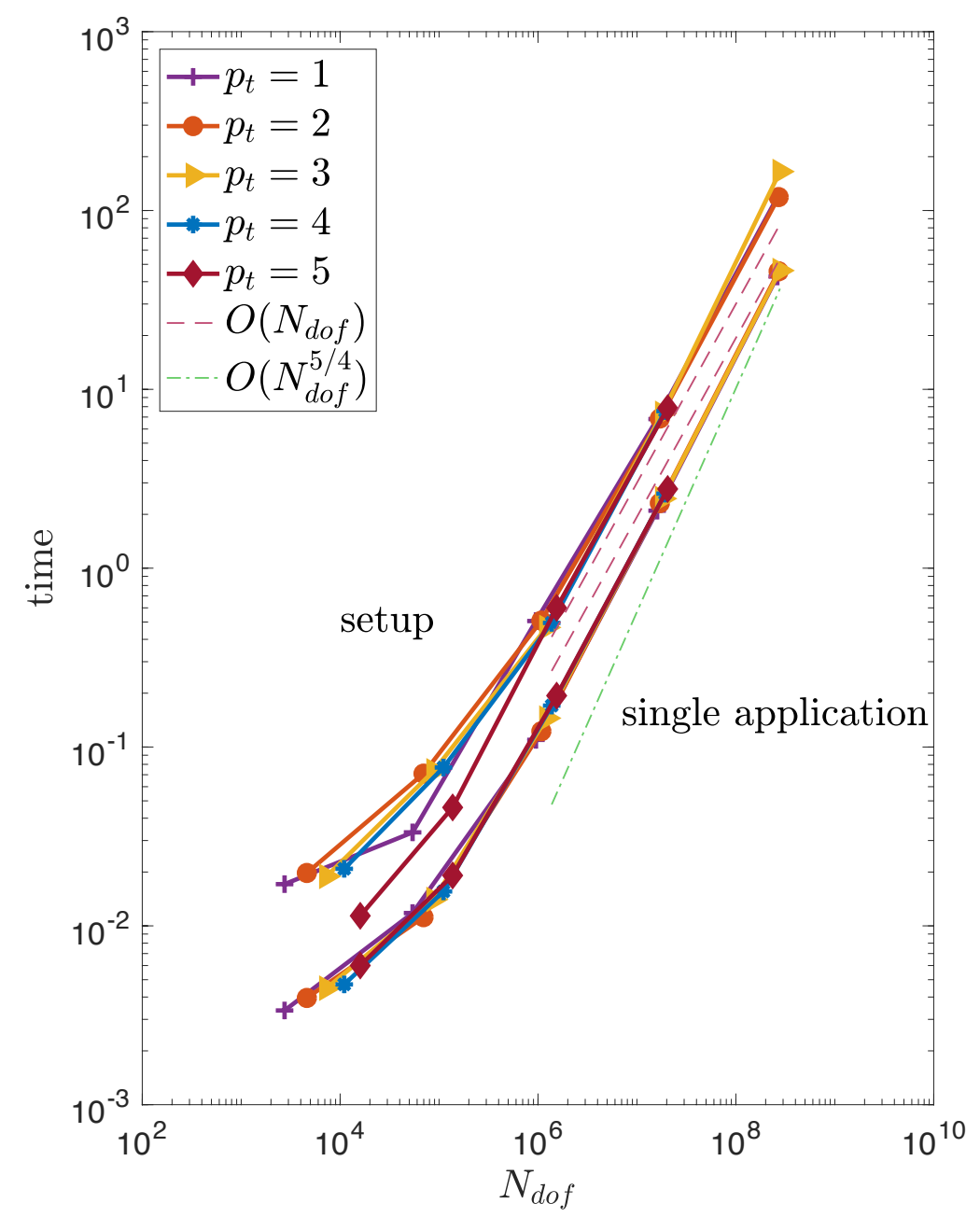

Figure 3. Setup time and single application time of $\widehat{\mathbf{A}}^{G}$ in the rotated quarter domain. 


\begin{tabular}{|c|c|c|c|c|c|c|}
\hline & \multicolumn{6}{|c|}{$\widehat{\mathbf{A}}$ Iterations / Time } \\
\hline$n_{e l}$ & $p=1$ & $p=2$ & $p=3$ & & $=4$ & $p=5$ \\
\hline 8 & $32 /$ & 70 / & $101 /$ & $128 /$ & 5.83 & $156 /$ \\
\hline 16 & $98 /$ & $121 / 10.54$ & $149 / 26.13$ & $167 /$ & 57.27 & $177 / 128.68$ \\
\hline 32 & $143 / 122.28$ & $165 / 236.47$ & $177 / 400.79$ & $193 /$ & 746.28 & $197 / 1230.60$ \\
\hline 64 & $165 / 3657.33$ & $168 / 4733.98$ & $175 / 6596.99$ & $179 /$ & 5894.01 & $184 / 20215.23$ \\
\hline
\end{tabular}

\begin{tabular}{|c|c|c|c|c|c|}
\hline & \multicolumn{5}{|c|}{$\widehat{\mathbf{A}}^{G} \quad$ Iterations / Time } \\
\hline$n_{e l}$ & $p=1$ & $p=2$ & $p=3$ & $p=4$ & $p=5$ \\
\hline 8 & $14 / \quad 0.30$ & $15 / \quad 0.50$ & $19 / 0.71$ & $20 /$ & $23 /$ \\
\hline 16 & $18 / 0.87$ & $19 / 1.66$ & $21 / 2.79$ & $23 /$ & $25 / \quad 14.12$ \\
\hline 32 & $22 / 8.88$ & $24 / 16.08$ & $25 / 29.66$ & $26 / 61.22$ & $27 / 114.93$ \\
\hline 64 & $26 / 207.70$ & $27 / 303.33$ & $28 / 495.29$ & $29 / 1118.44$ & $30 / 1923.20$ \\
\hline
\end{tabular}

Table 6. Hollow torus domain. Performance of $\widehat{\mathbf{A}}$ and $\widehat{\mathbf{A}}^{G}$.

\subsection{Performance of the preconditioner: hollow torus}

We consider a torus with a hole (Figure $1 \mathrm{~b}$ ) that is obtained by revolving an eccentric annulus (Figure 1c along the $x_{2}$-axis. For this problem we consider $\gamma=1$ and a separable in spatial and time variables, non-constant diffusion coefficient $\nu(\boldsymbol{x}, t)=\nu_{s}(\boldsymbol{x}) \nu_{t}(t)$. Precisely, we choose

$$
\nu_{s}(\boldsymbol{x}):=1+\frac{99}{2}\left[1+\frac{1}{\left(1+\frac{x_{1}^{2}}{x_{3}^{2}}\right)^{\frac{1}{2}}}\right] \text { and } \nu_{t}(t):=1+50\left[1+\cos \left(\frac{t}{2 \pi}\right)\right] .
$$

We remark that we are in the setting described in Section 4.4.1. The initial data and right-hand side are defined such that

$$
u(\boldsymbol{x}, t):=\sin \left(\pi x_{1}\right) \sin \left(\pi x_{2}\right) \sin \left(\pi x_{3}\right) \sin (\pi t)
$$

is the exact solution. In this case, we replace $\nu$ in 4.1 with its integral mean $\frac{1}{T|\Omega|} \int_{0}^{T} \int_{\Omega} \nu(\boldsymbol{x}, t) \mathrm{d} \Omega \mathrm{dt}$. In Table 6 we compare the performance of $\widehat{\mathbf{A}}$ (upper table) and $\widehat{\mathbf{A}}^{\boldsymbol{G}}$ (lower table): the inclusion of the information about the geometry parametrization and of the variable coefficient significantly reduces the number of iterations and the computational times.

\section{Conclusions}

In this work we proposed a preconditioner suited for a space-time Galerkin isogeometric discretization of the heat equation. Our preconditioner $\widehat{\mathbf{A}}$ is represented by a suitable sum of Kronecker products of matrices, that makes the computational cost of its construction (setup) and application, as well as the storage cost, very appealing. In particular the application of the preconditioner, inspired by the FD technique, exploits an ad-hoc factorization of the time matrices. The preconditioner cost seen in numerical tests, for a serial single core execution, is almost equal to $O\left(N_{\text {dof }}\right)$ and does not depend on the polynomial degree.

At the same time, the storage cost is roughly the same that we would have by discretizing separately in space and in time, if we assume $n_{t} \leq C p^{d} N_{s}$. Indeed, in this case the memory used for the whole iterative solver is $O\left(p^{d} N_{s}+N_{d o f}\right)$. 
In this paper, we have restricted ourselves to the case of a fixed domain and of constant (or separable) coefficients. However, the proposed approach can be extended to the case where the domain changes over time and/or the coefficients of the equation are not separable. Clearly, in these cases the matrix $\mathbf{A}$ is no longer the sum of Kronecker products as in (3.9), and its storage is likely unfeasible in practical problems. A possible way to circumvent this issue is to switch to a matrix-free approach 5, where the matrix is not stored and is available only to compute matrix-vector products. To build the preconditioner, the integral kernels that appear in the matrix entries should be replaced by separable approximations. This can be done using the same technique described in Section 4.4, at the (optimal) cost of $O\left(N_{d o f}\right)$ flops. A similar approach can be used if we consider a nonlinear problem, where a linear system of the form (3.8) has to be solved at each step of a nonlinear iteration. Note that in this case the preconditioner has to be build from scratch every time, as the matrix A changes at every iteration. This, however, is not an issue, since as discussed in Section 4.5 the setup cost for the preconditioner is optimal (or even negligible) and independent of $p$.

As a final comment, we mention that our method has a strong potential for parallelization, and this will be an interesting future direction of study.

\section{Acknowledgments}

The authors were partially supported by the European Research Council through the FP7 Ideas Consolidator Grant HIGEOM n.616563. The authors are members of the Gruppo Nazionale Calcolo ScientificoIstituto Nazionale di Alta Matematica (GNCS-INDAM) and the second author was partially supported by INDAM-GNCS "Finanziamento Giovani Ricercatori 2019-20" for the project "Efficiente risoluzione dell'equazione di Navier-Stokes in ambito isogeometrico". These supports are gratefully acknowledged.

\section{References}

[1] T. J. R. Hughes, J. A. Cottrell, Y. Bazilevs, Isogeometric analysis: CAD, finite elements, NURBS, exact geometry and mesh refinement, Computer Methods in Applied Mechanics and Engineering 194 (39) (2005) 4135-4195.

[2] J. A. Cottrell, T. J. R. Hughes, Y. Bazilevs, Isogeometric analysis: toward integration of CAD and FEA, John Wiley \& Sons, 2009.

[3] J. A. Evans, Y. Bazilevs, I. Babuška, T. J. R. Hughes, $n$-widths, sup-infs, and optimality ratios for the $k$-version of the isogeometic finite element method, Computer Methods in Applied Mechanics and Engineering 198 (2009) $1726-1741$.

[4] A. Bressan, E. Sande, Approximation in FEM, DG and IGA: a theoretical comparison, Numerische Mathematik (2019).

[5] G. Sangalli, M. Tani, Matrix-free weighted quadrature for a computationally efficient isogeometric $k$-method, Computer Methods in Applied Mechanics and Engineering 338 (2018) 117 - 133

[6] I. Fried, Finite-element analysis of time-dependent phenomena., AIAA Journal 7 (6) (1969) 1170-1173.

[7] J. C. Bruch Jr., G. Zyvoloski, Transient two-dimensional heat conduction problems solved by the finite element method, International Journal for Numerical Methods in Engineering 8 (3) (1974) 481-494.

[8] J. T. Oden, A general theory of finite elements. II. Applications, International Journal for Numerical Methods in Engineering 1 (3) (1969) 247-259.

[9] F. Shakib, T. J. R. Hughes, A new finite element formulation for computational fluid dynamics: IX. Fourier analysis of space-time Galerkin/least-squares algorithms, Computer Methods in Applied Mechanics and Engineering 87 (1) (1991) $35-58$.

[10] U. Langer, S. E. Moore, M. Neumüller, Space-time isogeometric analysis of parabolic evolution problems, Computer Methods in Applied Mechanics and Engineering 306 (2016) 342 - 363.

[11] U. Langer, M. Neumüller, I. Toulopoulos, Multipatch space-time isogeometric analysis of parabolic diffusion problems, in: International Conference on Large-Scale Scientific Computing, Springer, 2017, pp. 21-32.

[12] K. Takizawa, T. E. Tezduyar, Space-time computation techniques with continuous representation in time (st-c), Computational Mechanics 53 (1) (2014) 91-99.

[13] Y. Ueda, N. Saito, Stability and error estimates for the successive-projection technique with B-splines in time, Journal of Computational and Applied Mathematics 358 (2019) 266 - 278.

[14] M. J. Gander, M. Neumüller, Analysis of a new space-time parallel multigrid algorithm for parabolic problems, SIAM Journal on Scientific Computing 38 (4) (2016) A2173-A2208.

[15] C. Hofer, U. Langer, M. Neumüller, R. Schneckenleitner, Parallel and robust preconditioning for space-time isogeometric analysis of parabolic evolution problems, SIAM Journal on Scientific Computing 41 (3) (2019) A1793-A1821.

[16] A. Mantzaflaris, F. Scholz, I. Toulopoulos, Low-rank space-time decoupled isogeometric analysis for parabolic problems with varying coefficients, Computational Methods in Applied Mathematics 19 (1) (2019) 123-136.

[17] J. Bonilla, S. Badia, Maximum-principle preserving space-time isogeometric analysis, Computer Methods in Applied Mechanics and Engineering 354 (2019) 422-440. 
[18] K. Takizawa, T. E. Tezduyar, Y. Otoguro, T. Terahara, T. Kuraishi, H. Hattori, Turbocharger flow computations with the space-time isogeometric analysis (ST-IGA), Computers \& Fluids 142 (2017) 15-20.

[19] K. Takizawa, T. E. Tezduyar, T. Terahara, Ram-air parachute structural and fluid mechanics computations with the space-time isogeometric analysis (ST-IGA), Computers \& Fluids 141 (2016) 191-200.

[20] K. Takizawa, T. E. Tezduyar, T. Terahara, T. Sasaki, Heart valve flow computation with the space-time slip interface topology change (ST-SI-TC) method and isogeometric analysis (IGA), in: Biomedical Technology, Springer, 2018, pp. 77-99.

[21] M. Montardini, M. Negri, G. Sangalli, M. Tani, Space-time least-squares isogeometric method and efficient solver for parabolic problems, Mathematics of Computation (accepted for publication) (2019).

[22] G. Sangalli, M. Tani, Isogeometric preconditioners based on fast solvers for the Sylvester equation, SIAM Journal on Scientific Computing 38 (6) (2016) A3644-A3671.

[23] O. Steinbach, Space-time finite element methods for parabolic problems, Computational Methods in Applied Mathematics 15 (4) (2015) 551-566.

[24] R. Stevenson, J. Westerdiep, Stability of Galerkin discretizations of a mixed space-time variational formulation of parabolic evolution equations, arXiv:1902.06279 (2019).

[25] R. E. Lynch, J. R. Rice, D. H. Thomas, Direct solution of partial difference equations by tensor product methods, Numerische Mathematik 6 (1) (1964) 185-199.

[26] C. A. Dorao, H. A. Jakobsen, A parallel time-space least-squares spectral element solver for incompressible flow problems, Applied Mathematics and Computation 185 (1) (2007) 45-58.

[27] M. J. Gander, 50 years of time parallel time integration, in: Multiple Shooting and Time Domain Decomposition Methods, Springer, 2015, pp. 69-113.

[28] A. M. Kvarving, E. M. Rønquist, A fast tensor-product solver for incompressible fluid flow in partially deformed threedimensional domains: Parallel implementation, Computers \& Fluids 52 (2011) 22-32.

[29] C. De Boor, A practical guide to splines (revised edition), Applied Mathematical Sciences, Springer, Berlin, 2001.

[30] T. G. Kolda, B. W. Bader, Tensor decompositions and applications, SIAM review 51 (3) (2009) $455-500$.

[31] L. C. Evans, Partial Differential equations, American Mathematical Society, Berlin, 2010.

[32] L. Beirão da Veiga, D. Cho, G. Sangalli, Anisotropic NURBS approximation in isogeometric analysis, Computer Methods in Applied Mechanics and Engineering 209 (2012) 1-11.

[33] M. O. Deville, P. F. Fischer, E. H. Mund, High-order methods for incompressible fluid flow, Cambridge University Press, 2002.

[34] K. P. S. Gahalaut, S. K. Tomar, C. Douglas, Condition number estimates for matrices arising in NURBS based isogeometric discretizations of elliptic partial differential equations, arXiv preprint arXiv:1406.6808 (2014).

[35] M. Montardini, G. Sangalli, M. Tani, Robust isogeometric preconditioners for the Stokes system based on the Fast Diagonalization method, Computer Methods in Applied Mechanics and Engineering 338 (2018) 162 - 185.

[36] R. Vázquez, A new design for the implementation of isogeometric analysis in Octave and Matlab: GeoPDEs 3.0, Computers \& Mathematics with Applications 72 (3) (2016) 523-554.

[37] L. Sorber, M. Van Barel, L. De Lathauwer, Tensorlab v2. 0, Available online, URL: www.tensorlab.net (2014). 